\title{
Graphene-based materials for tissue engineering
}

Su Ryon Shin ${ }^{1,2,3 \dagger}$, Yi-Chen $\mathrm{Li}^{1,2,3 \dagger}$, HaeLin Jang ${ }^{1,2}$, Parastoo Khoshakhlagh ${ }^{1,2,3}$, Mohsen Akbari $^{1,2}$, Amir Nasajpour ${ }^{1,2}$, Yu Shrike Zhang ${ }^{1,2,3}$, Ali Tamayol ${ }^{1,2,3}$, Ali Khademhosseini ${ }^{1,2,3,4,5 *}$

$\left.{ }^{\dagger}\right]$ S.R. Shin and Y.C. Li contributed equally to this work.

[*]Corresponding authors: A. Khademhosseini

${ }^{1}$ Biomaterials Innovation Research Center, Department of Medicine, Brigham and Women's Hospital, Harvard Medical School, Cambridge, MA 02139, USA

${ }^{2}$ Harvard-MIT Division of Health Sciences and Technology, Massachusetts Institute of Technology, Cambridge, MA 02139, USA

${ }^{3}$ Wyss Institute for Biologically Inspired Engineering, Harvard University, Boston, MA 02115, USA

${ }^{4}$ Department of Physics, King Abdulaziz University, Jeddah 21569, Saudi Arabia.

${ }^{5}$ College of Animal Bioscience and Technology, Department of Bioindustrial Technologies, Konkuk University, Hwayang-dong, Kwangjin-gu, Seoul 143-701, Republic of Korea

Correspondence should be addressed to:

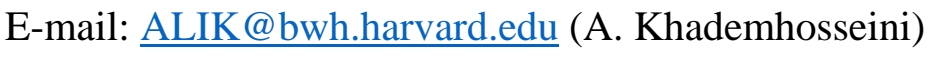

Keywords: Graphene, graphene oxide, graphene-based materials, tissue engineering, and regenerative medicine. 


\begin{abstract}
Graphene and its chemical derivatives have been a pivotal new class of nanomaterials and a model system for quantum behavior. The material's excellent electrical conductivity, biocompatibility, surface area and thermal properties are of much interest to the scientific community. Two dimensional graphene materials have been widely used in various biomedical research areas such as bioelectronics, imaging, drug delivery, and tissue engineering. In this review we will highlight the recent applications of graphene-based materials in tissue engineering and regenerative medicine. In particular, we will discuss the application of graphene-based materials in cardiac, neural, bone, cartilage, skeletal muscle, and skin/adipose tissue engineering. We also discuss the potential risk factors of graphenebased materials in tissue engineering. In addition, we will outline the opportunities in the usage of graphene-based materials for clinical applications.
\end{abstract}

\title{
Contents
}

1. Introduction

2. Properties of graphene and its chemical derivatives ..................................................

3. Graphene-based materials for tissue engineering applications.................................... 5

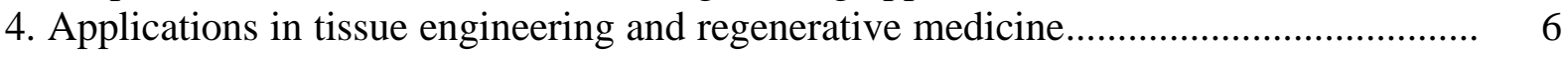

4.1 Cardiac tissue engineering and regeneration ....................................................... 6

4.2 Neural tissue engineering and regeneration ................................................... 8

4.2.1 Neuronal differentiation of stem cells on graphene …............................. 9

4.2.2 Neuronal activity on graphene ................................................................ 10

4.2.3 2D and 3D graphene constructs for neural regeneration ........................... 11

4.3 Bone tissue engineering and regeneration ........................................................ 12

4.3.1 Enhanced mechanical property by graphene ............................................ 14

4.3.2 Protein adsorption on graphene constructs ................................................. 15

4.4 Cartilage tissue engineering and regeneration ............................................. 15

4.5 Skeletal muscle tissue engineering and regeneration ....................................... 16

4.6 Skin/Adipose tissue engineering and regeneration ......................................... 17

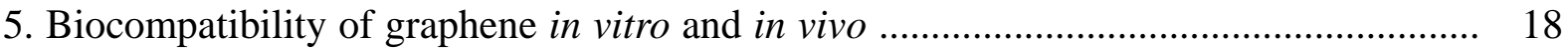

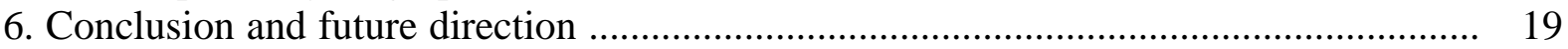

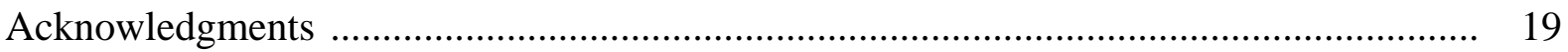

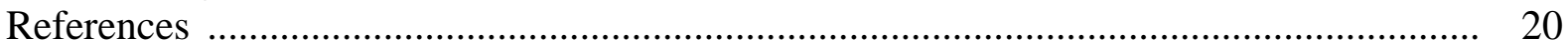

\section{Introduction}

Graphene is one of the crystalline forms of carbon, which is a single monolayer of $s p^{2}$ hybridized orbitals in a tightly packed to a two-dimensional (2D) honeycomb lattice (Fig. 1) [1]. Each carbon atom has three $\sigma$-bonds and an out-of-plane $\pi$-bond that can bind with neighboring atoms [1]. This atomic structure, combined with the electron distribution of graphene, results in high thermal electrical conductivity, unique optical behaviors, excellent mechanical properties, extreme chemical stability, and a large surface area. Furthermore, by chemical and physical modifications, graphene sheets can be transformed into graphenerelated materials such as single and multi-layered graphene, graphene oxide (GO), and reduced GO (rGO), each of which has unique tunable properties. Since its experimental discovery in 2004 [2] it has been widely used in various fields such as flexible electronics [3, 
4], supercapacitor [5], batteries [6], printable inks [7], optical, electrochemical sensors [8-10], and energy storage [11-13].

The field of tissue engineering involves the reproduction and regeneration of damaged tissues and organs $[14,15]$. To achieve its potential, engineered tissues require effective organization of cellular, morphological, and physiological features resembling those in vivo [16-18]. Therefore, the development of novel substitutes to create functional threedimensional (3D) complex tissue constructs in vitro or regenerate damaged tissues in vivo are of great importance. To engineer functional tissues, the generated substitutes have to possess several key factors such as guiding cell growth and modulation, delivering bioactive molecules, generating proper physical and chemical signal cues, and stimulating mechanical properties of the native tissue. These factors can be aided by incorporating specific nanomaterials that possess the unique features and properties in between single atoms and continuous bulk structure.

Graphene is one of the most versatile nanomaterials due to its exceptional physical and chemical properties. Moreover, graphene can interact with other biomolecules such as DNA, enzymes, proteins, or peptides for regenerative medicine and tissue engineering. In recent years, following the discovery of many interesting properties and biocompatibility, graphene and its chemical derivatives have emerged as a new class of nanomaterials and have been widely used in biomedical applications [19]. For example, while the excellent optical properties are suitable for bioimaging, graphene's large surface area and availability of free $\pi$ electrons are useful for gene and drug delivery platforms [20-27]. In addition, outstanding mechanical strength, stiffness and electrical conductivity make graphene-based materials a good candidate for bone and neural tissue engineering [13, 28-32].

In this review, we will highlight the recent applications of graphene-based materials in tissue engineering and regenerative medicine. In particular, we will emphasize the application of graphene-based materials in cardiac, neural, bone, cartilage, musculoskeletal, and skin/adipose tissue engineering. Although there is a great enthusiasm in using graphene-based materials in this field, there are still concerns regarding the potential toxicity and biocompatibility of these materials among the scientific and public communities. Therefore, we critically discuss this concern and propose potential solutions.

\section{Properties of graphene and its chemical derivatives.}

Graphene can be produced by various approaches including chemical vapor deposition [33], mechanical cleavage of graphite [34], and electrochemical exfoliation of graphite [35]. Graphene can be further wrapped up to form zero dimension (0D) nanomaterial like fullerenes, or rolled into nanotube (one dimension, 1D), or manipulated into 3D graphite [36]. Graphene sheets exist in bi-layers and multi-layers $(<10)$, each possessing unique properties. Further increasing the number of layers significantly changes the properties of the material as a graphene stack with 10 layers behave more like graphite [36], demonstrating that the interlayer structure and coupling between the layers determine key physical properties [37]. For instance, the stacking order, relative twist, and interlayer spacing govern the electronic, optical, and mechanical properties of multi-layered graphene [38-40].

Additionally, graphene is a free-standing two-dimensionally active carbon allotrope, where each carbon atom in the 2D crystal is bonded to the three other adjacent carbon atoms forming a hexagonal aromatic structure [1]. This specific structure and its periodicity result in unique electrical and mechanical characteristics. The electron confinement in the orbitals are localized to neighboring carbon atoms to create the covalent $\sigma$-bonds [41, 42]. The $\sigma$-bonds (C-C bonds) are responsible for the perfectly planar nature of graphene and determine its 
strong mechanical properties. Graphene's unique physical properties include hardness higher than diamond [43], elastic modulus as high as $1 \mathrm{TPa}$ [43], thermal conductivity almost 13 times higher than copper [44], and good optical transparency with $~ 97.7 \%$ transmittance [45]. Therefore, graphene can be blended with other materials such as synthetic or natural polymers to reinforce their mechanical and electrical properties. Moreover, the high surface area also causes other orbital electrons overlap and create $\pi$-planar densities on both sides for generating a $\pi$-bond between each pair of adjacent carbon atoms [46]. Graphene can also be easily functionalized with bioactive compounds such as proteins, enzymes, drugs, growth factors, and DNA $[47,48]$ via physical interaction. In addition, a large quality of bioactive compounds can be loaded on the systems large surface area $\left(2630 \mathrm{~m}^{2} / \mathrm{g}\right)$, as well as tuned to release the bioactive compounds in a controlled fashion via chemical-physical modification. Hence, based on those characteristics, many of the graphene-based drug/gene delivery systems such as nanocomposites of GO with vascular endothelial growth factor (VEGF) [22] or GO carrier containing mitoxantrone have been developed as cardiac tissue regeneration systems or a therapeutic treatment for tumors [49].

As a result of the $\pi$-bond structure and high surface area, the electron mobility of graphene is greater than $15,000 \mathrm{~cm}^{2} /(\mathrm{V} \cdot \mathrm{s})$ at room temperature [50], which is higher than the values observed in other materials such as carbon nanotubes (CNTs) or monocrystalline silicon currently used in electronics [34]. Additionally, this global $\pi$-configuration can be exploited by n-type [51] and p-type doping [52] agents to further tailor the electrical properties of graphene. For example, the electrical conduction of graphene decreases after doping with ethanol or ammonia and can be recovered when dopants are removed [53]. Thus, graphene might be an excellent material to stimulate and enhance the performance of electroactive cells between the biointerface and cellular membrane.

GO is a graphene derivative and the GO sheets that can be separated by treating graphite with potassium permanganate and sulfuric acid [54]. The structure of GO consists of a single atom layer and functional groups such as carboxylic acid, epoxide, and hydroxyl groups which could make it amphiphilic [55,56]. In particular, the carboxylic acid groups on the GO plane surface provide a colloidal stability and a $\mathrm{pH}$-dependent negative charge [57]. These functional groups (oxygen-containing) with different wettability as well as the large aromatic ( $\pi$-configuration) interface also endow GO with the ability to interact with DNA, peptides, or proteins via physical adsorption or chemical bonding. For instance, the thiolated DNA-coated GO could be subsequently used as a 2D bionano-interface for a homogeneous and stable assembly of gold $(\mathrm{Au}) /$ carbon structures [58]. Moreover, proteins or peptides, such as avidin or diphenylalanine (FF) peptides have also been used to functionalize graphene or GO [5961].

To increase the electrical conductivity properties of water soluble GO, rGO could be produced by reducing GO through thermal, chemical, or UV exposure processes. rGO has been generated by treatment with hydrazine at $100^{\circ} \mathrm{C}$ for 24 hours [62]. The generated rGO had a reduced amount of surface oxygens, making it less water stable and hydrophobic due to the fact that hydrazine was eliminated and pyrolyzed [63] However, hydrazine is a highly toxic reducing agent which is not suitable for biomedical applications and therefore a biocompatible reducing agent is required to create $\mathrm{rGO}$. In one study, ascorbic acid reduced rGO was tested versus the hydrazine method and it was evident that the ascorbic acid reduction method was more biocompatible than the hydrazine reduction methods[64]. For example, cells cultured on the collagen scaffold coated with rGO reduced by ascorbic acid possessed high cell viability and nontoxicity properties [65]. This indicates that ascorbic acid is a suitable reducing agent for $\mathrm{rGO}$ for biomedical applications. Notably, rGO has greater 
electron mobility (> $400 \mathrm{~S} / \mathrm{cm}$ ) [66]. rGO has been a fruitful material for engineering at the bio-nanointerface with neural and muscular tissues that require proper electrical properties to facilitate cell-cell signaling. Overall, graphene may be structurally and chemically modified to give an emergent platform for many biomedical applications.

\section{Graphene-based materials for tissue engineering applications}

Tissue engineering is a multi-disciplinary field that utilizes expertise in medicine, biology, and engineering to develop biomimetic tissue constructs for organ transplantation as well as for diagnostic and therapeutic research [16-18]. Biomaterials are crucial components in tissue engineering as they can induce specific cellular functions, direct cell differentiation, and modulate cell-cell interactions [67, 68]. However, different tissues in the body possess different mechanical, electrical, or physical properties. Single materials might not mimic the physical and biological properties of the native tissue; therefore, hybrid materials with multiple components that can address different requirements are widely used to fabricate artificial tissues. The extraordinary mechanical and electronic properties of graphene-based materials [69] have motivated researchers to use them in tissue engineering and regenerative medicine (Fig. 1) [13, 20, 21, 32]. Graphene can be combined with a variety of bioactive materials to engineer materials with desired characteristics.

Graphene has been demonstrated to adsorb nucleobases via $\pi-\pi$ interaction [70] and also effectively protect nucleotides from enzymatic cleavage [71]. Recently, gene therapy has become an important method for treating diseases in regenerative medicine. The basic requirements of a gene delivery vector include protecting DNA from degradation and ensuring high transfection efficiency (Fig. 2 (a)) [24]. Besides, non-viral and viral vectors also have been widely investigated for gene delivery research $[72,73]$. Therefore, graphene nanosheets may be suitable as a vector and that could be easily uptaken by cells. For instance, Chen et al. used a poly(ethylenimine)-GO (PEI-GO) carrier to transfect the plasmid DNA into HeLa cells and showed that PEI-GO could enhance the transfection efficiency by a proton-sponge effect [74].

Many researchers have employed various methods such as coating, hydrogel blending, wet/dry-spinning procedures, and 3D printing to make 2D or 3D graphene-based constructs. Graphene and its derivatives can also be tethered with other biomaterials to enhance their mechanical, physical, and electrical properties for tissue engineering applications. In our previous study, we showed that by using different concentrations of GO, the modulus of the GO-hybrid gels could be tunable within a range from 8-24 kPa [13]. Additionally, it is known that a biomaterial's surface properties, such as topography and stiffness, can regulate cellular behaviors, including adhesion or differentiation. It has been demonstrated that when a layer of graphene is coated on an $\mathrm{SiO}_{2}$ surface, it encourages stretching and proliferation of human mesenchymal stem cells (hMSCs) [75]. Moreover, graphene has been incorporated into other materials such as poly(L-lysine) (PLL) to significantly decrease their electrical resistance and stimulate the adhesion and neurite outgrowth of neurons [76].

Artificial fibrous structures which allow better nutrient transport are widely utilized in biomedical applications [77, 78]. Compared with composite fibers, graphene-based fibers show higher conductivity. $\mathrm{Xu}$ et al. and Jalili et al. provided two similar wet-spinning processes for making GO fibers by the liquid crystalline feature of GO (Fig. 2 (b)) [79-81]. In order to fabricate GO with a controlled diameter, researchers injected GO solution in coagulation baths that contained $\mathrm{NaOH}$ (aqueous solution), $\mathrm{KOH}$ or $\mathrm{CaCl}_{2}$ (in ethanol/water solution). Using the wet-spinning method a conductivity of more than $3.0 \times 10^{4} \mathrm{~S} / \mathrm{m}$ was achieved. This fibrous structure can be utilized as biofunctional scaffolds in skeletal tissue 
regeneration.

Recently, 3D printing techniques have been widely applied to tissue engineering due to the high precision 3D scaffold fabrication. Enhancement of mechanical properties and improvement in thermal and electrical conductivity have increased the demand to print graphene-based materials. Several studies have utilized composites of polymers including poly(ethylene glycol)-based copolymer or poly(vinyl alcohol) (PVA) and graphene or its derivatives to fabricate printable inks [82, 83]. Addition of GO to the polymer suspensions provides a shear-thinning behavior. However, studies have shown that the electrical conductivity of the printed constructs was limited due to the presence of the insulating polymer system $\left(<1.3 \times 10^{-2} \mathrm{~S} / \mathrm{m}\right)$ [83]. Liu et al., who created rGO fibers by a dry-spinning method, also prepared pure printable graphene inks in order to increase the electrical conductivity (Fig. 2 (c)) [66]. According to the mild thermal annealing behavior of GO [84], dispersed GO in water can be annealed to form a soft GO gel which can be printed to form a meshed fabric structure. The printed GO mesh has shown electrical conductivity as high as $6.6 \times 10^{4} \mathrm{~S} / \mathrm{m}$. This 3D structure formation of graphene-based materials can be used in cardiac or neural research areas. For example, Jakus et al. have developed a method to prepare a GO-based ink with high proportion of GO (more than 50\%) within poly(lactide-coglycolide) (PLG) [85]. In addition, cells on the printed construct showed high viability. Using this method, a complicated 3D architecture with high electrical conductivity and robust mechanical properties can be easily fabricated. Therefore, these novel 3D printed constructs have a great potential for use in regenerative medicine and tissue engineering when conductivity is needed.

\section{Applications in tissue engineering and regenerative medicine 4.1 Cardiac tissue engineering and regeneration}

Cardiac tissue injuries resulting from myocardial infarction or coronary artery diseases are among the most prevalent causes of morbidity and mortality around the globe. Loss of blood supply of the myocardium due to the occlusion of coronary arteries induces functional and morphological problems. Ischemia results in cardiomyocytes death through necrotic or apoptotic processes and scar tissue generation that alters contractile and electrophysiological characteristics. Since cardiac tissue is limited in its innate regenerative capacity, the damage is nearly permanent [86]. Current long-term strategy for management of cardiac injury relies on heart transplantation. However, since the number of donors is far less than the patient population, only a small fraction may receive new hearts. As a result, research efforts have been undertaken to develop approaches that can restore the biological and physical characteristics of the damaged tissue. Various novel therapeutic approaches have been introduced for heart regeneration including injecting stem cells, growth factors, peptides and biomaterials into the myocardium and generation of in vitro engineered cardiac patches in order to replace sections of damaged myocardial tissue [87]. However, the success of these patches has been limited by the challenge of creating engineered tissues that can reestablish the structure and function of the native cardiac tissue across different size scales [88].

Cardiac tissue possesses specific contractile properties that are directly related to its highly organized and anisotropic micro architecture $[89,90]$. Therefore, the proper function of engineered tissues and patches requires mimicking the anisotropic structure of the native myocardium, which can be achieved using a series of biophysical and topographical features. Additionally, cardiac muscles are electrically conductive (0.005 (transverse) $\sim 0.1$ (longitudinal) $\mathrm{S} / \mathrm{m}$ ). The unique mechanical properties of myocardium is a result of highly aligned collagen nanofibers with diameters ranging from 10 to $100 \mathrm{~nm}[89,91]$. Such 
nanoscale features play a vital role in regulating cellular functions.

To engineer electrically conductive materials, researchers have doped biomaterials with electrically conductive nanomaterials such as gold and carbon-based nanoparticles [90]. Such materials have been used for creating 2D or 3D scaffolds for cardiac tissue engineering and have shown improvements in cellular function. The improved cellular performance correlates with enhanced material stiffness and conductivity that lead to improved cell-cell signaling through the nanoparticles. Following these observations, nanomaterials have also been injected in injury sites to improve healing process. However, challenges such as the retention of the implanted nanocarriers at the site of injury, particularly in the dynamic beating myocardium environment, hampers the success of the therapy. This challenge has been addressed by incorporating nanocarriers within the matrix of injectable hydrogels [92].

Stem cells possess excellent therapeutic applications for the repair and regeneration of injured myocardium. Preliminary studies demonstrated that stem cells may enhance myocardial perfusion and contractile functionality in the injured heart through differentiation into cardiomyocytes or vascular specific cellular phenotype. Cytoprotective factors released from stem cells have been shown to promote myocyte survival and reduce inflammatory responses. Stem cells are also heavily influenced by multitude of microenvironmental cues (i.e. biophysical and biochemical). Graphene and its derivatives could be used to control the fate of stem cells in vivo by providing morphological cues as well as enhancing the electrical conductivity of the matrix.

Recent studies have demonstrated the ability of graphene to stimulate the cardiomyogenic differentiation in MSCs via simply culturing MSCs on the graphene monolayers [93, 94]. After MSCs were co-implanted with GO flakes, their survival rate improved significantly as GO protected the cells from reactive oxygen species in vivo (Fig. 3 (a)) [95]. Incorporation of rGO flakes within hMSC spheroids was shown to alter the expression of cell signaling molecules and improve the expression of cardiac specific markers [96]. The enhanced behavior of cardiac spheroids might be due to the high affinity of rGO flakes towards fibronectin along with high conductivity. In a mouse model of myocardial infarction (MI), the implantation of hybrid rGO-hMSC spheroids improved cardiac repair and function when compared to either hMSCs or rGO alone. Furthermore, significantly enhanced therapeutic efficacy (10 folds lower dose of MSCs) was achieved through implantation of hybrid rGO-MSC spheroids [96]. The main advantages of such delivery methods are that the nanoparticles and cells can be accurately placed at the site of injury and the drug can be delivered in a more sustained manner. Graphene-based materials have further been incorporated within hydrogel scaffolds to improve their mechanical properties and electrical conductivity. GO/gelatin methacryloyl (GelMA) hybrid hydrogel constructs with tunable mechanical and electrical conductivities were fabricated to create 3D engineered tissues (Fig. 3 (b) ) $[13,32$, 97]. GO was preferred over graphene as it is more water soluble due to its oxygen rich surface which facilitates in creating a more dispersed aqueous solutions[13]. GelMA has allowed recapitulating the complex structures by using photopatterning approaches. Moreover, the GO sheets were readily suspended and uniformly dispersed in GelMA pre-polymer solution, which is likely facilitated by the strong non-covalent interaction between GO and GelMA [13]. The mechanical property of hybrid hydrogels containing GO/GelMA was enhanced by covalently conjugating GO into the hydrogel matrix by introducing methacryloyl functional groups onto GO [32]. Additionally, covalent conjugation of GO within GelMA enabled dispersing higher concentrations of GO $(3 \mathrm{mg} / \mathrm{ml})$ without aggregation or significant increase in the viscosity [32].

In a recent study, our group has engineered a 3D cardiac tissue multi-array based on a 
systematic layer-by-layer assembly of cells within GO-based films (Fig. 3 (b)) [97]. The GO film acted as an adhesive layer that ensured strong adhesion of different layers. This strategy enabled the creation of multi-layer functional tissue construct from cardiomyocytes and endothelial cells. The thickness of the fabricated tissue was controlled by adjusting the quantity of the deposited GO. Three cell types of hMSCs, cardiomyocytes and endothelial cells were incorporated into the construct by using this approach. The results indicated that the existence of the GO-based film improved cardiac cell maturation, cell-cell electrical coupling, and organization. This was demonstrated by upregulation of cardiac markers, such as sarcomeric $\alpha$-actinin, and strong beating of the construct under low external electric fields. In another study, we added GO to methacryloyl-substituted recombinant human tropoelastin (MeTro) to develop an elastic and electrically conductive biomaterial for cardiac tissue engineering (Fig. 3 (c)) [98]. The addition of GO to the MeTro matrix improved its elastic modulus by $53 \%$ and rupture strain by $45 \%$. Moreover, the MeTro/GO gel exhibited remarkable resistance against rotation stress without any plastic deformation as it did not exhibit any noticeable hysteria after $23 \pm 5$ rotations while pristine MeTro gels deformed after the first few rounds of rotation and were ruptured after $15 \pm 4$ rotations. Addition of GO nanoparticles to the MeTro gel increased its electrical conductivity and resulted in a nonuniform surface topography. These changes in the material properties improved the function of cardiomyocytes seeded on the MeTro/GO gel sheets. It was observed that the average area covered by cells was increased twice in the presence of pristine MeTro after one day of culture. Furthermore, the expression of cardiac markers such as sarcomeric $\alpha$-actinin, connexin-43, and troponin I was higher than pure MeTro and was improved when the concentration of GO increased.

Overall, graphene-based materials hold great promise for engineering functional cardiac tissues. Most hydrogels used for cardiac tissue engineering are mechanically weaker than natural tissue. The addition of graphene-based nanoparticles can improve the mechanical and surface properties of these materials in several ways. Moreover, these nanoparticles increase the electrical conductivity of inherently insulating hydrogels, which can enhance cell-cell signaling and improve signal propagation that are important for cardiac tissue engineering. Addition of graphene-based materials to hydrogels provide nanotopographies similar to those experienced by stem cells in vivo. Therefore, these materials can be used in stem cell-based therapies to direct their fate, but some limitation such as biocompatibility needs to be resolved in the future.

\subsection{Neural tissue engineering and regeneration}

The nervous system is a network of neural lineage cells that transmit related signals to synchronize the brain and parts of the body. Neurons are electrically excitable cells that maintain a voltage gradient across their membranes by using ion pumps that generate concentration gradient differences of ions such as potassium, calcium, chloride, and sodium. Neurons transmit electrical signals by utilizing chemical transmitters such as acetylcholine via a specialized synapse structure [99]. An additional unique property of neurons is that they lack the ability to undergo mitosis, and therefore enhancing the ability of stem cells to differentiate into neurons provides an attractive therapeutic strategy for the treatment of neuronal diseases. The conductive nature of graphene, [100] as well as its biocompatibility, makes it a great candidate to recapitulate the intricate signaling process associated with the nervous system.

\subsubsection{Neuronal differentiation of stem cells on graphene}


The combination of embryonic stem cells (ESCs) and graphene has been fruitful in the sense that it poses a favorable environment for pluripotent cells to differentiate into neurons [101]. Yang et al. compared the neuronal differentiation ability of ESCs on graphene and GO [102]. They found that, in a study of ESCs cultured on GO substrates, dopaminergic neuronal differentiation was twice more than that on graphene. They also confirmed that dopaminergic neuron differentiation of ESCs could be regulated by the concentration of GO. In particular, the number of differentiated dopaminergic neurons at the highest tested concentration of GO $(100 \mu \mathrm{g} / \mathrm{ml})$ was increased three times than the lowest concentration of $\mathrm{GO}(1 \mu \mathrm{g} / \mathrm{ml})$. Wang et al. cultured MSCs on a substrate of functionalized fluorinated graphene and demonstrated neuronal differentiation. They suggest that the strong polarity of carbon-fluorine bonds has triggered biological responses such as improving metabolic activities and strengthening the interaction between drugs and a targeted protein [103]. In addition, the strong polarity of fluorine as well as the high protein adsorption [104] led to MSCs differentiation into neuronal lineages.

Neural stem/precursor cells (NSCs) have been widely used to repair neuronal diseases by injection of exogenous NSCs in animal models $[105,106]$. For example, Dibajina et al. directly injected NSCs in the lateral ventricle, which improved recovery from brain ischemia [107]. Park et al. were the first to explore the fundamental effects of graphene-based materials on the behaviors of human NSCs (hNSCs) [108]. The researchers cultured hNSCs in a medium with growth factors combined with 2D graphene film where the hNSCs demonstrated a faster proliferation rate compared with the control group. In additon, when exposed to medium with no growth factors, cells expressed a neuronal specific marker (TUJ1) and differentiated neurons demonstrated an excellent neuronal activity on the graphene film.

Researchers have been able to utilize tunable materials in order to investigate mechanotransduction pathways along with upstream cytoskeletal and downstream gene expression factors [109-111]. In particular, neuronal outgrowth and differentiation is sensitive to surface biomolecules, morphologies, and roughness [112]. GO can be functionalized with different functional groups such as amino-, sulfonic acid-, or methoxyl-groups [113]. In addition, biomimetic acetylcholine- and phosphorylcholine-like units such as dimethylaminoethyl methacrylate (DMAEMA) and 2-methacryloyloxyethyl phosphorylcholine (MPC) can be immobilized on the GO surface [114]. Therefore, neurite extension and the number of neuronal branches could be regulated by these functional groups on the GO substrate, especially on DMAEMA-bound GO. In addition, the electrical properties of graphene-based substrates may induce neuronal differentiation from stem cell. GO is also used to regulate the differentiation of NSCs when combined with other conductive polymers such as poly(3,4-ethylenedi-oxythiophene) (PEDOT) or poly(pyrrole) [115]. It is difficult to manipulate the nano- and microscale morphology of traditional conductive polymers. Thus, Weaver et al. synthesized a GO/PEDOT nanocomposite in order to create a conductive film with high roughness that may enhance neuronal differentiation [48]. In this paper, GO/PEDOT films showed high roughness with microsheet-like features compared to PEDOT/PSS films. This indicates that GO could provide tunable properties to control surface morphologies of smooth substrates. Subsequently, GO-PEDOT could be easily functionalized with interferon- $\gamma$ (IFN $\gamma$ ) by chemical crosslinking due to presence of free functional groups on the GO surface (Fig. 4 (a)). Researchers have also demonstrated that neuronal differentiation was improved on the IFN $\gamma$-crosslinked GO-PEDOT film (IFN $\gamma$-CL) and neurons migrated out from the cellular clusters. To successfully achieve neuronal regeneration, proper differentiation of both neurons and oligodendrocytes is necessary [116]. Oligodendrocytes on GO-coated poly(caprolactone) (PCL) substrates could be differentiated 
up to five times compared to that of pristine PCL substrate, which implies that GO promotes oligodendrocyte differentiation.

During the regenerative process, neurites should interconnect and regain electrical continuity [117]. Lorenzoni et al. have confirmed that patterned single layer graphene substrate can promote neurite outgrowth to form an organized interconnected neuronal network on the graphene [118]. In addition, neuronal differentiation due to GO has been confirmed by Solanki et al. They demonstrated that a GO-coated silica nanoparticle monolayer enhanced hNSCs differentiation and induced excellent axonal alignment [119]. Lorenzoni and Solanki et al. demonstrated that improved neurite outgrowth, neuronal networking, and axonal alignment can result from proteins such as poly(D-lysine) or laminin adsorbed on the pattern of graphene-based materials. Furthermore, the GO substrate could maintain the high cellular viability over a prolonged period after differentiation. This particular feature has benefits for regenerative medicine and tissue engineering applications such as stem cell differentiation and survival. Numerous studies have demonstrated GO can efficiently guide the differentiation of stem cells due to various characteristics of GO including amphiphilicity and surface chemistry [120-123]. To control stem cell fate, Kim et al. developed a concept for regulating human adipose-derived stem cells (ADSCs) differentiation combined with patterns of GO [124]. They utilized a photolithographic technique to generate two different types of nano-GO (NGO) patterns (GO line and GO grid patterns), and then cultured hADSCs on GO patterns. Neuronal differentiation from hADSCs is considered a challenge as it implied a conversion from mesoderm to ectoderm [125]. Thus, an NGO grid hybrid pattern was selected as the geometry to mimic the in vivo environment that had elongated and interconnected structure. It is assumed that the NGO grid pattern might enhance electrical signaling of neuronal network [126]. Interestingly, the morphology of hADSCs cultured on NGO grid hybrid revealed an internetwork-like formation after 5 days of incubation (Fig. 4 (b)). In contrast, hADSCs on PLL-Au, NGO-Au substrates expressed a random arrangement. Furthermore, after a long-term culture, a distinct change in cellular morphology was observed. hADSCs on an Au substrate had a circular morphology and cells on the NGO-Au grid pattern revealed a bipolar orientation with cellular extensions. Additionally, cells on the NGO grid pattern also expressed high degree of neurite extension and growth. The expression of specific neuronal marker (TUJ1) evidenced the NGO-Au grid pattern led to a higher neuronal differentiation of inducted capacity than Au or NGO-coated Au substrate.

\subsubsection{Neuronal activity on graphene}

Graphene-based materials have been applied in neural interface and electrical recording because of their excellent electrical properties [127, 128]. Therefore, the combination of graphene and neuroscience to develop an advanced neuronal interface has become a new strategy for enhancing the record of neuronal signal or stimulating the neuronal activity [129]. The effect of graphene electrodes on neuronal activity of differentiated neurons was estimated in a recent study [130]. After formation of the neuronal network, an approximate $30 \%$ increase in neuronal signaling was observed in the network formed on graphene films and spontaneous $\mathrm{Ca}^{2+}$ oscillations of neuron rose to twice that of the control group. These results indicated that graphene-based materials might support the growth of functional neural circuits and enhance the activity of neuronal networks. Liu et al. developed an implantable graphene-based neural electrode to detect the electrophysiological and neurochemical signaling in vivo [131]. They presented an $\mathrm{rGO} / \mathrm{Au}_{2} \mathrm{O}_{3}$ nanocomposite-coated electrode to detect the concentration of $\mathrm{H}_{2} \mathrm{O}_{2}$ in an in vivo hyperacute stroke model. This rGO-modified 
electrode provided high $\mathrm{H}_{2} \mathrm{O}_{2}$ sensitivity, low detection limits, and stronger electron transfer between tissue and electrode interfaces than traditional gold electrodes. Furthermore, Feng et al. presented soft graphene-based nanofibers for detection of neuronal activity [132]. First, the negatively charged GO sheet was assembled on $\mathrm{NH}_{3}$ plasma treated poly(vinyl chloride) nanofibers (called GO-NFs), and the GO-NFs were reduced to obtain the graphene nanofiber (G-NFs). The ultrathin graphene shell of G-NFs, which wrapped the nanofiber surface tightly, resulted in high electrical conductivity and excellent flexibility. Subsequently, compared with the neurons on graphene film (G-film) and tissue culture plate (TCP), neurons on the G-NFs expressed more branches of neurites and a faster growth rate. In addition, on the G-NFs, neurons exhibited high levels of TUJ1 expression as well as microtubule-associated proten-2 (MAP or MAP2) (maturated neuronal marker) caused by the geometry cues of G-NFs, which closely resemble nanofibers (Fig. 4 (c-i)). In particular, by adding electrical stimulation on neurons through G-NFs (called G-NFs/ES), a noticeable accelerated growth rate that was nearly twice of that of neurons on the G-NFs was observed. This promotion can be due to the induction of $\mathrm{Ca}^{+}$influx, which activates the calmodulin-kinases pathways due to the depolarizing effect of the G-NFs activated voltage-gated $\mathrm{Ca}^{2+}$ channels in order to increase the intercellular $\mathrm{Ca}^{2+}$ concentration (Fig. 4 (c-ii)). Also, Feng et al. indicated that the 3D GNF nanofibers have a great impact on the development and growth of motor neurons. G-NFs provide an anisotropic electron transfer which can lead to a localized electrical stimulation at the contact points of neurons. Regulation of $\mathrm{Ca}^{2+}$ and the excellent electrical conductive properties of graphene-based materials make them great candidates for neuronal applications.

\subsubsection{D and 3D graphene constructs for neural regeneration}

For nerve injuries, autologous nerve grafting by suture is a "gold standard" for small damaged nerve defects/gaps in clinics [133]. Axonal regeneration has a slow regeneration rate (about 2-5 mm/day) [134, 135] which results in obstacles for the treatment of long defects/gaps. To this end, an appropriate supporting bridge, conduit, to correctly guide axon growth to reconnect damaged nerves is necessary. Hong et al. reported that a 2D graphene film grown on catalytic copper by chemical vapor deposition method not only had good biocompatibility but also simulated the neurite outgrowth, indicating that the graphene-based material was a suitable candidate for repairing injured nerves [136]. Additionally, many reports also have indicated that external electrical fields could promote neurite growth and direct the orientation of neurons $[137,138]$. In an example, Li et al. also cultured NSCs on 3D graphene foams (3D-GF) and observed an enhancement in neuronal differentiation [139]. GF is a 3D porous structure with a high specific surface area $\left(200-800 \mathrm{~m}^{2} / \mathrm{g}\right)$ that allows the growth of NSCs, and the interconnected pores also support the cellular communication and ensure the efficient mass transport of nutrients for cells. Moreover, 3D-GF possessed microscale topographic features such as curvatures or anisotropic microstructures, which was different from 2D graphene-based materials so that the neuronal differentiation might be enhanced by these various surface properties [76].

Although graphene-based materials have been widely utilized to fabricate films [131] or 3D scaffolds [132, 139], there is ongoing studies in order to extend the versatility and functionality of graphene and its chemical derivatives for neural regenerative medicine. 3D printing techniques might be a suitable method to fabricate the complex neural structure such as brain or neuronal conduit. Shah et al. mixed graphene and poly(lactide-co-glycolide) (PLG) as a bioink, and then fabricated a custom-sized nerve conduit by a 3D printing technique [85]. In their report, by mixing graphene with PLG as a 3D printable graphene (3DG) ink, they demonstrated that, via increasing the concentration of graphene from $20 \%$ to $60 \%$, the ranges 
of elastic strain are decreased from $210 \%$ to $81 \%$, and conductivity could be raised from 200 to $600 \mathrm{~S} / \mathrm{m}$, respectively. After this, 3DG was used to culture hMSCs, and a high proliferative behavior was observed after hMSCs cultured on 20\% and 60\% 3DG for 14 days. Meanwhile, the expressions of neuronal specific markers (TUJ1 and MAP2) of the cells were significantly increased after 14 days of differentiation. It was also confirmed that 3DG possessed a neuronal-induction effect on hMSCs under a simple growth medium without neurogenic stimuli (Fig. 5 (a)). Additionally, on 60\% 3DG printed scaffolds, high aspect ratio extension of neurons (more than $100 \mu \mathrm{m}$ ) could be observed. Notably, cells on 3DG scaffolds formed a wire-like network, which connected with individual cells (Fig. 5 (b-d)). Subsequently, upon closer inspection of cells, expression of uni- or multi-polar neuronal morphologies [140] such as the $2 \mu \mathrm{m}$-wide axon-like process and presynaptic terminals was evident (Fig. 5 (d)). Moreover, by applying the graphene-based conduit in a human cadaver model, the 3DG printed conduit (Fig. 5 (e) and (f)) was wrapped around the nerve bundle, sutured along the nerve conduit, and attached to the surround, epicedium and nerve bundle by sutures. This model indicated that the strong mechanical properties of the graphene-based conduit can be advantageous in surgical procedures (Fig. 5 (g)).

Overall, the positive role of graphene and its derivatives have been confirmed in electrical stimulation of neural cells for the growth, differentiation, and the development of neural lineage cells. Additionally, the tunable surface and machining properties of graphenebased materials are suitable to fabricate the neuronal tissue-like structures in order to align the arrangement of neurons. These properties suggest that graphene-based materials have great potentials for neural tissue engineering applications.

\subsection{Bone tissue engineering and regeneration}

To enhance bone regeneration by stimulating cellular adherence, proliferation, and osteoblastic differentiation, graphene-based materials are widely used alone or incorporated into bone implants and scaffolds [141]. It is reported that excellent mechanical strength and protein adsorption of graphene-based materials can enhance osteogenic differentiation of human MSCs [141-143]. Remarkably, graphene can accelerate cellular differentiation without commonly used additional bone growth factors, such as BMP-2, while stem cells did not differentiate in the absence of graphene [144]. This might be due to the fact that graphene can increase local dexamethasone concentration via $\pi$ - $\pi$ stacking between the aromatic rings in the biomolecules and the graphene basal plane which can enhance the osteogenic differentiation. [120, 145].

Incorporation of graphene and its chemical derivatives in materials used for bone regeneration can enhance osteoconductivity through stimulating both biomineralization and cellular osteogenic differentiation. For example, biominerals such as calcium carbonate $\left(\mathrm{CaCO}_{3}\right)$ mixed with graphene and $\mathrm{GO}$ sheets could augment biomineralization [146]. Also, osteoblasts grown on mineralized GO or graphene calcium phosphate composites exhibited high viability with elongated shape. When graphene hydrogel films were implanted in rat models, they induced bone regeneration by osteogenic differentiation of MSCs due to their rough surface morphology and excellent mechanical properties [147]. It implies that the high elastic modulus of graphene-based materials ( 1 to $2.4 \mathrm{TPa}$ ) can be a driving force for spontaneous osteogenic differentiation [148]. Disordered topography can provide better environments for protein adsorption and cellular growth. In addition, porous graphene hydrogels made by a non-covalent interaction have higher mechanical strength than conventional hydrogel systems with similar water content level (90\%) while simultaneously 
maintaining its mechanical flexibility [147]. When a graphene hydrogel film was implanted in a subcutaneous site, the hydrogel film remained stable. After 12 weeks from the implantation, abundant vascularization was observed around the graphene hydrogel film and there was no severe inflammatory response.

The inorganic part of bone is composed mostly of hydroxyapatite (HAP: $\left.\mathrm{Ca}_{10}\left(\mathrm{PO}_{4}\right)_{6}(\mathrm{OH})_{2}\right)$ which can support bone regeneration. Graphene-based materials have been combined with HAP to enhance osteogenic differentiation of cells and formation of new bone $[146,149,150]$. It was reported that $\mathrm{GO} / \mathrm{CaCO}_{3}$ or graphene/CaCO $\mathrm{CO}_{3}$ hybrid composites can enhance formation of HAP when incubated in simulated body fluid (SBF) solution [146]. Based on the convergence of graphene with biomineralization, the resulted GO/grapheneHAP composites demonstrated high viability of osteoblasts with elongated morphology, providing a microenvironment similar to in vivo. To enhance biomimetic mineralization on $\mathrm{GO}$, the surface of GO can be modified with functional groups, such as sulfate groups, to stimulate $\mathrm{Ca}^{2+}$ binding and thus provide nucleation points for HAP mineralization [151]. For example, carrageenan (Car), a natural polysaccharides consisting of highly sulfated units, can be used to functionalize the surface of graphene and induce HAP mineralization. Based on the excellent biocompatibility of Car-GO, MC3T3-E1 cells grown on Car-GO showed higher viability and proliferation level with elongated shapes compared to cells on GO or glass substrates. In addition, cellular ALP activity on Car-GO was significantly higher than GO or glass substrates. As a result, not only did Car-Go enhance the cellular attachment, but it also stimulated bone mineralization activity of cells. Once HAP-including vesicles were released from the osteoblast cells, ions will deposit and bone mimetic crystallites will grow.

Graphene and HAP nanoparticles can self-assemble into 3D nanocomposite hydrogel based on colloidal chemistry synthesis, using aqueous suspensions of GO nanosheets and citrate-stabilized HAP nanoparticles [152]. During hydrothermal treatment, thickness of GO nanosheets increases as they attract each other through $\pi-\pi$ interaction, based on the increased hydrophobicity. In addition, GO reduces into rGO due to the presence of citrate ions, since precursor ions that can be oxidized are reported to rGO [153, 154]. Once the graphite-like shell structure is developed, the shell functions as a dialysis membrane to remove excess amount of ions including sodium citrates and deposit destabilized HAP nanoparticles on graphene flasks. As a result, HAP nanoparticles become entrapped inside the 3D graphene network, forming homogenous graphene-HAP gel. When mouse MSCs were cultured on graphene-HAP nanocomposite hydrogels, high cellular viability was observed with more elongated morphology compare to rGO hydrogel, indicating enhanced cellular affinity on graphene-HAP nanocomposite hydrogel.

rGO sheets and HAP microparticles can adhere with each other through electrostatic interactions and hydrogen-bonding $[149,150]$. The positively charged calcium moieties on the surface of HAP microparticles can be immobilized on the negatively charged hydroxyl and carboxyl groups on the surface of rGO sheets via electrostatic interactions. From the zeta potential analysis, surface charges of rGO sheets and HAP microparticles in deionized (DI) water ( $\mathrm{pH} 7.0$ ) were measured to be around $-34.5 \mathrm{mV}$ and $+6.5 \mathrm{mV}$, respectively [150]. After rGO sheets partially covered HAP microparticles, the surface charge was measured to be around $-23.7 \mathrm{mV}$. In addition, the hydroxyl group of HAP microparticles and the oxygencontaining groups of rGO sheets can induce hydrogen-bonding interaction. As a result, HAP microparticles can be coated by the interconnected network of rGO.

Nanocomposites made by rGO sheets and HAP microparticles demonstrated enhanced cellular viability compared to HAP microparticles. Mitigated cytotoxicity of $\mathrm{rGO} / \mathrm{HAP}$ nanocomposite may be derived from the coating effect of rGO sheets on HAP particles, since 
cellular growths were inhibited under the higher concentration of rGO sheets $(>31.3 \mu \mathrm{g} / \mathrm{ml})$ compare to HAP microparticles $(>10 \mu \mathrm{g} / \mathrm{ml})$. rGO/HAP nanocomposite can also enhance spontaneous osteodifferentiation of preosteoblasts (MC3T3-E1) in vitro, with significant increase in expression levels of osteopontin and osteocalcin. In addition, the calcium deposition in MC3T3-E1 cells were significantly greater under the influence of rGO/HAP nanocomposite, compare to that of HAP (Fig. 6 (a)) [155]. Notably, rGO/HAP nanocomposite was able to induce calcium deposition in MC3T3-E1 without any osteogenic agents. When implanted into a critical-sized bone defect model, rGO/HAP nanocomposite reduced inflammatory responses and stimulated new bone formation (Fig. 6 (b)) [156].

Effects of graphene-based materials combined with other composites, such as strontium and calcium silicates, on osteogenic differentiation are also widely investigated $[157,158]$. When strontium particles are encapsulated in GO and $\mathrm{rGO}$, strontium ions can be continuously released from the composite scaffolds and stimulate cellular proliferation and their osteogenic differentiation [157]. In addition, when graphene is mixed with calcium silicate $\left(\mathrm{CaSiO}_{3}\right)$, which is already a known biomaterial to enhance osteoblastic differentiation, rGO added $\mathrm{CaSiO}_{3}$ matrix stimulated proliferation and ALP activities of human osteoblast cells better than calcium silicate ceramics, indicating synergetic effects of graphene-based materials and biocompatible materials[158].

\subsubsection{Enhanced mechanical property by graphene}

Graphene can reinforce the mechanical properties of hybrid materials for load-bearing implant applications to regenerate bone tissue. The toughening mechanism for graphenebased materials are based on crack bridging, pull-out, crack deflection, and crack tip shielding [159]. Graphene can bridge across the crack to provide closure stress to relax fracture stress. Also, graphene pulled out from the material can impede crack propagation by the interfacial friction between graphene and matrix material. When crack encounters graphene, it changes the direction of crack propagation that results energy dissipation and thus hinders crack propagation. In addition, crack tip can be blocked by graphene phase as additional energy is required for interface debonding.

Graphene-based materials can be used to improve mechanical strengths of bioceramics, hydrogels, and bioactive glasses, without damaging their structures or biocompatibilities [159]. In a graphene incorporated HAP system, it was reported that fracture toughness can be increased up to $56 \%$ with $4 \%$ of CNT in HAP coating [160]. In addition, elastic modulus, fracture toughness and wear resistance of CNT reinforced HAP composite fabricated by spark plasma sintering were increased up to $25 \%, 92 \%$, and $66 \%$ respectively, compare to pure HAP [161, 162]. Graphene-incorporated hydrogel film was reported to stimulate the calcium deposits and osteogenic differentiation of stem cells without any additional inducer, while host tissue response was observed at the minimum level in vivo at subcutaneous sites of rats [147]. Among many unique properties of graphene, osteogenic stimulation mechanism of the self-supporting graphene hydrogel was highly supported by its exceptional mechanical strength, as stiff substrates are known to promote bone differentiation [143]. Self-supporting graphene hydrogel had much higher tensile modulus of $69 \pm 5 \mathrm{MPa}$ [147], while conventional polymer hydrogels were reported to have tensile modulus range between 0.01 to $10 \mathrm{kPa}$ [163].

\subsubsection{Protein adsorption on graphene constructs}

Graphene is the strongest, thinnest, and lightest material, which can be used as substrates for biomolecules to enhance cellular attachment, growth and activity [36, 164]. Due to their high surface area, graphene materials can absorb a large amount of small molecules. For the graphene-family nanomaterials, including graphene, graphite, GO, and rGO, biomolecules 
with low solubility, partial hydrophobicity, or positive charge are generally favored for adsorption [165-167]. For rGO, proteins can be adsorbed on the surface through hydrophobic interactions and $\pi-\pi$ stacking interactions. Also, for GO the system exhibits $\pi-\pi$ stacking interactions, hydrophobic interaction, and hydrogen bonding between the nitrogen/oxygen containing groups of GO and oxygen functional groups in proteins are known to be involved during protein absorption. To enhance the amount of protein adsorption on graphene constructs, surfaces of graphene-based materials can be functionalized or modified to optimize their hydrophilicity/hydrophobicity to attract proteins with complex amphiphilic properties [141, 168-170].

As an example, surface oxygen content can affect protein adsorption level of graphenebased materials. Shi et al. used a low-temperature thermal reduction method to control oxygen content of GO which further optimized reduction states of multi-layer rGO (MrGO) [141]. After thermal reduction, increased amount of proteins, such as fibronectin, fibroblast growth factor, bovine serum albumin, and fetal bovine serum, were absorbed on the surface of $\mathrm{MrGO}$, through non-covalent interactions. As a result, cellular attachment, proliferation and bone-mineralization related activities were enhanced in cells grown on the MrGO. In addition, based on the protein adsorption capability, GO can be utilized for the delivery of bone morphogenetic protein-2 (BMP-2) and substance P (SP) through electrostatic interaction between the ionized groups of GO and proteins and hydrophobic $\pi-\pi$ stacking [171].

\subsection{Cartilage tissue engineering and regeneration}

Human cartilage tissue is composed of approximately $1 \%(\mathrm{v} / \mathrm{v})$ of highly specialized chondrocytes embedded within a pool of extracellular matrix proteins, such as collagens, glycosaminoglycan (GAG), proteoglycans, and noncollagenous proteins [172, 173]. Articular cartilage enables low-friction movement of synovial joints while exhibiting sufficient level of strong mechanical properties to sustain body weight. However, unlike other tissues such as bone, cartilage tissue is unable to spontaneously recover once damaged, as it is almost avascular and acellular. In this regard, cellular therapies using MSCs are widely used in clinics to regenerate cartilage tissues by inducing chondrogenic differentiations in defect sites. To induce the cellular repair process, graphene has been incorporated as a scaffold for the stem cell therapy of cartilage treatment, based on its stimulation effects on cellular growth/differentiation and excellent mechanical properties.

To deliver both cells and proteins for cartilage tissue engineering, graphene-based materials can act as "growth factor factories". Graphene/cell biocomposites can be formed by assembling MSCs with graphene flakes and act as a pre-concentrate platform for cartilage tissue engineering [173-175]. The schematic of the process for making graphene/cell biocomposite is shown in Fig. 7 (a) [176], which describes preparation of graphene-based solutions in fetal bovine serum and chondrogenic chemical inducers. After hMSCs were added into the graphene solution, as shown in Fig. 7 (b-f), the cells and graphene-based materials were self-assembled into a bio-composite. The nanoscale porosity of GO could be controlled by acid and base treatment after its synthesis to maximize its protein loading capacity. The resulting graphene-cell biocomposites could enhance cellular viability and their chondrogenic differentiation behaviors, which is expressed by high deposition of chondrocyte-specific extracellular matrix (aggrecan, collagen II, and GAG). Since the presence of GO in the composite can accelerate cellular chondrogenic differentiation, engendering porosity of graphene can increase loading amount of GO thus enhance cellular differentiation rate. Before incorporating into cellular pellets, GO sheets could also adsorb fibronectin (FN) and TGF- $\beta$ proteins through $\pi-\pi$ and electrostatic interaction while 
preserving protein structures to promote chondrogenic differentiation [174]. By incorporating GO sheets as protein delivery carriers, cellular pellets with sizes larger than their general diffusion limitation (150-200 $\mu \mathrm{m}$ ) became available to utilize, as chondrogenic inducers can be supplied to cells located inside the core region of cellular pellets [174].

Graphene-based materials can act as mechanical and electrical cues in cartilage scaffolds, as they can improve overall mechanical strength and conductivity. As shown in Fig. 7 (g), a hybrid cartilage scaffold composed of chondroitin sulfate methacryloyl, poly(ethylene glycol) methyl ether- $\varepsilon$-caprolactone-acryloyl chloride, and GO (CSMA/PECA/GO) was used to provide biomimetic 3D environment [175]. Cartilage cells grown on this CSMA/PECA/GO scaffold proliferated with high viability in vitro, indicating biocompatibility of the scaffold. When the CSMA/PECA/GO scaffolds were implanted in the osteochondral defect of a rabbit model, CSMA/PECA/GO scaffolds with cell injection induced higher volume of newly formed cartilage/bone tissues, compared to scaffold-only group [175].

\subsection{Skeletal muscle tissue engineering and regeneration}

As skeletal muscle tissues have limited regeneration capacities, once they are severely damaged, permanent loss of tissue function can occur. To regenerate skeletal muscle tissue, stem cell based therapies are often used with nanomaterials that can act as functional scaffolds to stimulate cellular viability and differentiation. In this respect, since graphenebased materials possess excellent flexibility, relatively high electrical conductivity $(0.6 \mathrm{~S} / \mathrm{m})$, and strong mechanical properties with ultralow density $\left(0.16 \mathrm{mg} / \mathrm{cm}^{3}\right)$, they can act as excellent cellular substrates for muscle tissue engineering [177]. Due to its excellent elastic stiffness, ultralight and highly compressible graphene aerogel, which was prepared by applying microwave reduction to the mixture of GO with ethylenediamine, could fully recover after $90 \%$ compression without fracturing, in the cyclic compression test. Even after 1000 times of compressions, graphene aerogel could maintain $70 \%$ of the maximum stress value and recover its original volume [177].

Graphene-based materials can enhance adhesion, proliferation, and myogenesis of skeletal muscle cells (Fig. 8) [178, 179]. When C2C12 myoblasts were grown on ultrathin thermally reduced (TR) graphene-based films, cells demonstrated higher attachment and better spreading behavior than GO and glass slide [178]. During in vitro cellular bioactivity evaluation, conductivity of TR-graphene substrate was more than 3 times of that of GO substrates. As a result, when electrical stimulation was applied, differentiation of C2C12 myoblasts into myotubes was accelerated on TR-graphene substrates compared to GO and glass slides, with increased myotube length and cell-covered area. Gene expression levels related to the contraction capabilities of cells and myotubes differentiation, such as $\alpha$-actinin, muscle regulatory factor 4, myosin heavy chain isofactorIId/x (MHC-IId/x), and sarcomeric actin, were significantly enhanced in cells grown on TR-graphene substrate than GO and glass slides. Interestingly, while $\mathrm{C} 2 \mathrm{C} 12$ cells grown on TR-graphene substrates showed significantly enhanced differentiation level under electrical stimulation, C2C12 cells grown on GO and glass slides only showed similar or marginal improvement in their differentiation level. For example, after electrical stimulation application, expression level of $\mathrm{MHC}-\mathrm{IId} / \mathrm{x}$ increased over 10-fold in $\mathrm{C} 2 \mathrm{C} 12$ cells grown on TR-graphene substrates, while expression level of MHC-IId/x did not show significant change in C2C12 cells grown on GO and glass slides.

Graphene-based materials can also enhance cellular alignment and thus maximize cellular contractile power for skeletal muscle tissues [179, 180]. Based on the attachment preference of $\mathrm{C} 2 \mathrm{C} 12$ cells on graphene over $\mathrm{SiO}_{2}$. Bajaj et al patterned graphene islands on 
oxide surfaces using lithography to induce highly aligned cells. Myotube area fractions on graphene and oxide surfaces were $76 \%$ and $7.4 \%$, respectively, and thus spontaneous alignment of myotubes was achieved on graphene patterns. When electrical stimulation was applied from the waveform generator, myotubes were contracted at the same rate with the applied frequency pulse.

\subsection{Skin/Adipose tissue engineering and regeneration}

Current tissue engineering solutions to skin regeneration are complicated by wound structure, secretion and formation of ECM. Materials with microporous structure and high surface-to-volume ratios similar to the ECM of tissue is considered beneficial to skin regeneration [181]. Graphene and GO can offer solutions to these problems, as they may be strong skin tissue engineering platforms due to their high surface area and protein adsorption properties. Researchers have shown that GO-poly(lactic-co-glycolic acid)/collagen (GOPLGA/collagen) hybrid fiber sheets with high hydrophilicity and surface energy could promote the adhesion of human dermal fibroblasts (HDFs). The use of collagen allowed biochemical interactions with the HDFs comparable to integrin-ECM interaction. This leads to the conclusion that $\mathrm{GO}$ and collagen may favorably interact to promote skin regeneration due to their combined advantageous impact on the proliferation and migration of dermal fibroblasts. The cells cultured on GO-PLGA/collagen sheets grew in a similar manner to what is typically seen on PLGA/collagen sheets with the added benefit of increased proliferation (Fig. 9 (a) and (b)) [181].

Li et al. demonstrated that GF loaded with MSCs enhanced wound healing while having strong biocompatibility. In this research GF promoted growth and proliferation of MSCs (Fig. 9 (c) and (d)), leading to improved wound closure in animal models that included thicker and more skin tissue growth two weeks after implantation (Fig. 9 (e-g)). In these models there was also an upregulation of VEGF and basic fibroblast growth factor (bFGF), both drivers of neovascularization. In addition, there might be an anti-scarring benefit as TGF- $\beta 1$ and alphasmooth muscle actin ( $\alpha$-SMA) were downregulated while TGF- $\beta 3$ was unregulated [182].

In some cases, graphene and GO have been shown to have strong noncovalent bindings with a variety of bioactive agents that improve stem cell proliferation, including MSCs. Lee et al. utilized graphene and GO sheets as a tissue engineering platform due to their high inplane stiffness and biocompatibility. They demonstrated that graphene and GO acted as preconcentration platform for stem cell inducers, leading to MSCs differentiation that can be attributed to their interactions with the graphene structure. Furthermore, GO was found to allow adipogenesis due to their electrostatic binding with insulin [120].

Other researchers described GO films coated on glass substrates, which were used to culture human ADSCs (hADSCs). The hADSCs demonstrated increased amounts of focal adhesions when grown on the GO films, along with increased correlation between actin filaments when compared to hADSCs grown on uncoated glass. The GO films also enhanced hASC differentiation, including osteogensis, adipogenesis and epithelial genesis compared to uncoated glass substrates [183].

\section{Biocompatibility of graphene in vitro and in vivo}

The use of graphene for biomedical applications has led to thorough analyses of its toxicity and biocompatibility. Similar to other carbon-based materials, graphene is nonbiodegradable and may lead to severe toxicity in areas such as the lung. Graphene demonstrates little biological degradation, though carboxylated derivatives may be tuned to degrade in the appropriate circumstances [184]. Studies have focused on a variety of methods to prepare graphene and the resulting cytotoxic effects. For example, graphene prepared 
through chemical vapor deposition increased apoptosis, level of lactate dehydrogenase, and the generation of reactive oxygen species in neural cells [185]. A level of $50 \mu \mathrm{g} / \mathrm{ml}$ of GO in fibroblasts has been demonstrated to result in significant cytotoxicity [186]. Others have demonstrated that a concentration of roughly $80 \mu \mathrm{g} / \mathrm{ml}$ may be the threshold for cytotoxicity in A549 human carcinomic alveolar basal epithelial cells (A549 cells), as apoptosis at higher levels of exposure may be possible.

In vivo toxicity of graphene has also been demonstrated in animal models such as the lungs of rats and mice, where injection of GO led to dose-dependent toxicity through accumulation over long periods of time [187]. These toxic effects of GO may be attributed to GO being unstable in vivo and the non-specific binding characteristics of proteins to GO. In this case, GO was captured in the lung, as it is the initial organ the graphene-derived material was carried after injection. In another study, it was found that GO had a longer blood circulation time than comparable nanomaterials while having low uptake into the reticuloendothelial system [188]. While investigating a $1 \mathrm{mg} / \mathrm{kg}$ body weight dose of GO over a 14-day period, researchers found no pathological changes in the organs which were examined along with strong biocompatibility with red blood cells. Another study evaluated the thrombogenic properties of a scaffold that was surface modified with rGO. When compared to the non-coated cellular tissue, there was no significant difference in platelet adhesion between the two. Furthermore, no adherent platelet-leukocyte aggregates were observed and there was no difference in DNA fragmentation. These results indicated that rGO is a strong material for modifying bioprosthetic heart valves due to its hemocompatibility [189].

As mentioned above, graphene and its derivative materials can be modified in order to improve its biocompatibility. For example, Wang et al. used a bottom-up approach to synthesize amine-functionalized graphene quantum dots (GQDs), which resulted in watersoluble single-crystalline GQDs with strong absorption bands within the visible region. This material demonstrated a high cellular viability and low toxicity level over time (over $90 \%$ in $48 \mathrm{hrs}$ ) compared to traditional quantum dots (Fig. 10) [190]. Also, graphene/ PCL composites were synthesized by the ring-opening polymerization of $\varepsilon$-caprolactone under the exposure of GO nanosheets. After culturing the fibroblasts on the nanosheets, a significant increase in cell density on graphene/PCL was observed over 4 days that demonstrated equally strong biocompatibility [191]. Additionally, upon PEGylation, similarly, graphene may have far better biocompatibility and behave differently in vivo. Presence of PEGylated graphene in sizes of $10-50 \mathrm{~nm}$ led to a minimal cytotoxicity over a month-long period. The PEGylated graphene accumulated in the liver and spleen instead of the lung, and was gradually excreted over time [192]. Lu et al. further demonstrated in an in vivo study that PEG-functionalized 125I-labeled nanographene sheets (NGS) exhibited reduced toxicity. In a long-term toxicity study of a $20 \mathrm{mg} / \mathrm{kg}$ dose, mice biochemistry, hematology and histology were examined for a 3-month period. After IV administration, PEGylated NGS was found to accumulate in the reticuloendothelial system, principally in the liver and the spleen, and was gradually excreted, demonstrating strong biocompatibility due to fictionalization [193]. Moreover, another study investigated the biocompatibility of GO among two levels of oxygenation following subcutaneous and peritoneal tissue implantation in order to investigate GO's influence on medical devices (Fig. 11) [194]. An inflammatory response consistent with normal foreign body reaction was demonstrated in these studies. $20 \mathrm{mg} / \mathrm{kg}$ of GO with different $\mathrm{C}$ to $\mathrm{O}$ ratios and were injected into tissue sites. The presence of inflammatory cells was quantified utilizing flow cytometry and compared to a PBS injection control. Macrophage levels were monitored over a two-week period, and the study demonstrated that macrophage levels were 
lower in the GO groups than control. Notably, higher oxidation levels of GO resulted in increased numbers of monocytes and an enhanced pro-inflammatory environment was identified. To sum up, we realize that the major parameters that can cause toxicity in graphene-based materials are the dose, size, time of exposure, and number of layers of graphene. In particular, the surface properties of graphene and its derivatives such as functional group or chemical structure were considered as highly related with their toxicity. The cytotoxicity was reduced by functionalizing the derivatives with biocompatible compounds or controlling the size of the compounds using synthetic methods. Additionally, graphene-based materials may also generate oxidative debris and induce cytotoxicity. Thus, the purity of graphene and its derivatives during functionalization should be considered. Overall, due to various sizes, shapes, and fabrication of graphene-based materials, there is still a need to standardize the validation of toxicity for clinical usage in the future.

\section{Conclusions and future direction}

Significant advances have been made during the past decade in the development of graphene-based materials. Recently, graphene-based materials have been found to have many applications in the fields of tissue engineering and regenerative medicine due to their ability to provide excellent physical, chemical, and biological properties. The peculiar atomic arrangements endow graphene with a strong adsorbing capacity for use as gene/drug delivery vehicles. Graphene and its derivatives also provide a new selection for enhancing the mechanical and surface properties of biomaterials to regulate the cardiomyogenic, neurogenic, osteogenic and cartilaginous abilities of stem cells. Additionally, the high electrical conductivity of graphene and its derivatives is expected to stimulate the growth and activities of electrically excitable cells such as cardiomyocytes and neurons, and enhancing the record of the cellular signals. Subsequently, coupling the tunable surface chemical and machinable properties for 3D printing technology further creates complex graphene architectures and efficiently extends their applications. Moreover, combination of graphene and its derivatives with other compositions that have properties such as wettability or flexibility make them good candidates to fabricate multi-functional smart materials. As a result, the graphene composites can become environmentally sensitive or can possess shape-memory or selffolding properties which can extend their biomedical applications. As an example, GO mixed with a phase changing material was utilized to create a thermo-responsive drug delivery system. This system improves drug-loading efficiency along with photothermal effects for photodynamic therapies. Also, combining graphene with polydopamine can create a selffolding mobile device. Furthermore, combining the self-folding graphene-based materials and 3D printing technology will help develop the next generation of printing techniques to apply in tissue engineering. Although, graphene and its derivatives have been widely used for these ranges of biomedical applications, problems still exist when it comes across the cytotoxic or genotoxic effects of these nanomaterials. There is not a gold standard to overcome the biocompatibility issues associated with graphene products in living systems. This necessitates establishing graphene-based models to evaluate and control cellular behaviors in vitro. Surface modification including protein or cytokine functionalization has attracted a great deal of attention to resolve the biocompatibility concerns. Further developing the testing model to understand the mechanisms of graphene-based materials cytotoxicity is worth deeper investigation before graphene and its derivatives may be eventually applied to the clinics.

\section{Acknowledgements}

This paper was supported by National Science Foundation (EFRI-1240443), ONR PECASE 
Award, and the National Institutes of Health (HL092836, DE019024, EB012597, AR057837, DE021468, HL099073, EB008392).

\section{Reference}

[1] A.K. Geim, Graphene: status and prospects, Science, 324 (2009) 1530-1534.

[2] K.S. Novoselov, A.K. Geim, S. Morozov, D. Jiang, Y. Zhang, S.a. Dubonos, I. Grigorieva, A. Firsov, Electric field effect in atomically thin carbon films, Science, 306 (2004) 666-669.

[3] G. Eda, G. Fanchini, M. Chhowalla, Large-area ultrathin films of reduced graphene oxide as a transparent and flexible electronic material, Nat. Nano, 3 (2008) 270-274.

[4] I. Meric, M.Y. Han, A.F. Young, B. Ozyilmaz, P. Kim, K.L. Shepard, Current saturation in zero-bandgap, top-gated graphene field-effect transistors, Nature nanotechnology, 3 (2008) 654-659.

[5] S. Sahoo, P. Bhattacharya, S. Dhibar, G. Hatui, T. Das, C.K. Das, Graphene/poly(anilineco-pyrrole) nanocomposite: potential candidate for supercapacitor and microwave absorbing applications, J. Nanosci. Nanotechnol., 15 (2015) 6931-6941.

[6] M.K. Dufficy, S.A. Khan, P. Fedkiw, Hierarchical graphene-containing carbon nanofibers for lithium-ion battery anodes, ACS Appl. Mater. Interfaces, (2015).

[7] C. Zhu, T.Y. Han, E.B. Duoss, A.M. Golobic, J.D. Kuntz, C.M. Spadaccini, M.A. Worsley, Highly compressible 3D periodic graphene aerogel microlattices, Nat. Commun., 6 (2015) 6962.

[8] Y. Shao, J. Wang, H. Wu, J. Liu, I.A. Aksay, Y. Lin, Graphene based electrochemical sensors and biosensors: a review, Electroanalysis, 22 (2010) 1027-1036.

[9] M. Pumera, A. Ambrosi, A. Bonanni, E.L.K. Chng, H.L. Poh, Graphene for electrochemical sensing and biosensing, TrAC Trends in Analytical Chemistry, 29 (2010) 954-965.

[10] W. Yang, K.R. Ratinac, S.P. Ringer, P. Thordarson, J.J. Gooding, F. Braet, Carbon nanomaterials in biosensors: should you use nanotubes or graphene?, Angew.e Chem. Int. Ed., 49 (2010) 2114-2138.

[11] M. Pumera, Graphene-based nanomaterials for energy storage, Energy Environ.1 Sci., 4 (2011) 668-674.

[12] M.F. El-Kady, R.B. Kaner, Scalable fabrication of high-power graphene microsupercapacitors for flexible and on-chip energy storage, Nat. Commun., 4 (2013) 1475.

[13] S.R. Shin, B. Aghaei-Ghareh-Bolagh, T.T. Dang, S.N. Topkaya, X. Gao, S.Y. Yang, S.M. Jung, J.H. Oh, M.R. Dokmeci, X.S. Tang, A. Khademhosseini, Cell-laden microengineered and mechanically tunable hybrid hydrogels of gelatin and graphene oxide, Adv. Mater., 25 (2013) 6385-6391.

[14] R. Langer, J. Vacanti, Advances in tissue engineering, J. Pediatr. Surg., (2015).

[15] M.J. Webber, O.F. Khan, S.A. Sydlik, B.C. Tang, R. Langer, A perspective on the clinical translation of scaffolds for tissue engineering, Ann. Biomed. Eng., 43 (2015) 641-656.

[16] A. Khademhosseini, J.P. Vacanti, R. Langer, Progress in tissue engineering, Sci. Amer., 300 (2009) 64-71.

[17] R. Langer, Biodegradable polymer scaffolds for tissue engineering, Nat. Biotechnol., (1994).

[18] A. Tamayol, M. Akbari, N. Annabi, A. Paul, A. Khademhosseini, D. Juncker, Fiber-based tissue engineering: Progress, challenges, and opportunities, Biotechnol. Adv., 31 (2013) 669687. 
[19] C. Chung, Y.K. Kim, D. Shin, S.R. Ryoo, B.H. Hong, D.H. Min, Biomedical applications of graphene and graphene oxide, Acc. Chem. Res., 46 (2013) 2211-2224.

[20] X. Sun, Z. Liu, K. Welsher, J.T. Robinson, A. Goodwin, S. Zaric, H. Dai, Nano-graphene oxide for cellular imaging and drug delivery, Nano Res., 1 (2008) 203-212.

[21] C. Wang, J. Li, C. Amatore, Y. Chen, H. Jiang, X.M. Wang, Gold nanoclusters and graphene nanocomposites for drug delivery and imaging of cancer cells, Angew. Chem. Int. Ed. Engl., 50 (2011) 11644-11648.

[22] A. Paul, A. Hasan, H.A. Kindi, A.K. Gaharwar, V.T. Rao, M. Nikkhah, S.R. Shin, D. Krafft, M.R. Dokmeci, D. Shum-Tim, A. Khademhosseini, Injectable graphene oxide/hydrogel-based angiogenic gene delivery system for vasculogenesis and cardiac repair, ACS Nano, 8 (2014) 8050-8062.

[23] M. Nurunnabi, K. Parvez, M. Nafiujjaman, V. Revuri, H.A. Khan, X. Feng, Y.-k. Lee, Bioapplication of graphene oxide derivatives: drug/gene delivery, imaging, polymeric modification, toxicology, therapeutics and challenges, RSC Adv., 5 (2015) 42141-42161.

[24] S. Goenka, V. Sant, S. Sant, Graphene-based nanomaterials for drug delivery and tissue engineering, J Control Release, 173 (2014) 75-88.

[25] J.S. D. Depan, R.D.K. Misra,, Controlled release of drug from folate-decorated and graphene mediated drug delivery system: Synthesis, loading efficiency, and drug release response, Mater. Sci. Eng. C, 31 (2011) 1305-1312.

[26] H.S. Jung, M.-Y. Lee, W.H. Kong, I.H. Do, S.K. Hahn, Nano graphene oxide-hyaluronic acid conjugate for target specifc cancer drug delivery, RSC Adv., 4 (2014) 14197-14200.

[27] S.H. Hu, Y.W. Chen, W.T. Hung, I.W. Chen, S.Y. Chen, Quantum-dot-tagged reduced graphene oxide nanocomposites for bright fluorescence bioimaging and photothermal therapy monitored in situ, Adv. Mater., 24 (2012) 1748-1754.

[28] A. Schinwald, F.A. Murphy, A. Jones, W. MacNee, K. Donaldson, Graphene-based nanoplatelets: a new risk to the respiratory system as a consequence of their unusual aerodynamic properties, ACS Nano, 6 (2012) 736-746.

[29] X. Yang, L. Qiu, C. Cheng, Y. Wu, Z.F. Ma, D. Li, Ordered gelation of chemically converted graphene for next-generation electroconductive hydrogel films, Angew. Chem. Int. Ed. Engl., 50 (2011) 7325-7328.

[30] L. Zhang, Z. Wang, C. Xu, Y. Li, J. Gao, W. Wang, Y. Liu, High strength graphene oxide/polyvinyl alcohol composite hydrogels, J. Mater. Chem., 21 (2011) 10399-10406.

[31] J. Shen, B. Yan, T. Li, Y. Long, N. Li, M. Ye, Mechanical, thermal and swelling properties of poly (acrylic acid)-graphene oxide composite hydrogels, Soft Matter., 8 (2012) 1831-1836.

[32] C. Cha, S.R. Shin, X. Gao, N. Annabi, M.R. Dokmeci, X.S. Tang, A. Khademhosseini, Controlling mechanical properties of cell-laden hydrogels by covalent incorporation of graphene oxide, Small, 10 (2014) 514-523.

[33] N.G. Shang, P. Papakonstantinou, M. McMullan, M. Chu, A. Stamboulis, A. Potenza, S.S. Dhesi, H. Marchetto, Catalyst-free efficient growth, orientation and biosensing properties of multilayer graphene nanoflake films with sharp edge planes, Adv. Funct. Mater., 18 (2008) 3506-3514.

[34] K.S. Novoselov, A.K. Geim, S.V. Morozov, D. Jiang, Y. Zhang, S.V. Dubonos, I.V. Grigorieva, A.A. Firsov, Electric field effect in atomically thin carbon films, Science, 306 (2004) 666-669.

[35] N. Liu, F. Luo, H. Wu, Y. Liu, C. Zhang, J. Chen, One-step ionic-liquid-assisted electrochemical synthesis of ionic-liquid-functionalized graphene sheets directly from graphite, Advanced Funct. Mater., 18 (2008) 1518-1525. 
[36] A.K. Geim, K.S. Novoselov, The rise of graphene, Nat. mater., 6 (2007) 183-191.

[37] L. Brown, R. Hovden, P. Huang, M. Wojcik, D.A. Muller, J. Park, Twinning and twisting of tri-and bilayer graphene, Nano Lett., 12 (2012) 1609-1615.

[38] A. Luican, G. Li, A. Reina, J. Kong, R.R. Nair, K.S. Novoselov, A.K. Geim, E.Y. Andrei, Single-layer behavior and its breakdown in twisted graphene layers, Phys. Rev. Lett., 106 (2011) 126802.

[39] S. Shallcross, S. Sharma, W. Landgraf, O. Pankratov, Electronic structure of graphene twist stacks, Phys. Rev. B, 83 (2011) 153402.

[40] E. Suárez Morell, P. Vargas, L. Chico, L. Brey, Charge redistribution and interlayer coupling in twisted bilayer graphene under electric fields, Phys. Rev. B, 84 (2011) 195421.

[41] D.E. Jiang, B.G. Sumpter, S. Dai, Unique chemical reactivity of a graphene nanoribbon's zigzag edge, J. Chem. Phys., 126 (2007) 134701.

[42] C. Wu, S.D. Sarma, px,y-orbital counterpart of graphene: Cold atoms in the honeycomb optical lattice, Phys. Rev. B, 77 (2008) 235107-235113.

[43] C. Lee, X. Wei, J.W. Kysar, J. Hone, Measurement of the elastic properties and intrinsic strength of monolayer graphene, Science, 321 (2008) 385-388.

[44] A.A. Balandin, S. Ghosh, W. Bao, I. Calizo, D. Teweldebrhan, F. Miao, C.N. Lau, Superior thermal conductivity of single-layer graphene, Nano Lett., 8 (2008) 902-907.

[45] D.E. Sheehy, J. Schmalian, Optical transparency of graphene as determined by the finestructure constant, Phys. Rev. B, 80 (2009) 193411.

[46] X. Gao, Y. Zhao, B. Liu, H. Xiang, S.B. Zhang, Pi-Bond maximization of graphene in hydrogen addition reactions, Nanoscale, 4 (2012) 1171-1176.

[47] Z. Liu, B. Liu, J. Ding, J. Liu, Fluorescent sensors using DNA-functionalized graphene oxide, Anal Bioanal Chem, 406 (2014) 6885-6902.

[48] C.L. Weaver, X.T. Cui, Directed neural stem cell differentiation with a functionalized graphene oxide nanocomposite, Adv. Healthc. Mater., 4 (2015) 1408-1416.

[49] L. Hou, Q. Feng, Y. Wang, X. Yang, J. Ren, Y. Shi, X. Shan, Y. Yuan, Z. Zhang, Multifunctional hyaluronic acid modified graphene oxide loaded with mitoxantrone for overcoming drug resistance in cancer, Nanotechnology, 27 (2016) 015701.

[50] C. Gomez-Navarro, R.T. Weitz, A.M. Bittner, M. Scolari, A. Mews, M. Burghard, K. Kern, Electronic transport properties of individual chemically reduced graphene oxide sheets, Nano Lett., 7 (2007) 3499-3503.

[51] S.Y. Zhou, D.A. Siegel, A.V. Fedorov, A. Lanzara, Metal to insulator transition in epitaxial graphene induced by molecular doping, Phys. Rev. Lett., 101 (2008) 086402.

[52] J.-H. Chen, C. Jang, S. Adam, M.S. Fuhrer, E.D. Williams, M. Ishigami, Chargedimpurity scattering in graphene, Nat. Phys., 4 (2008) 377-381.

[53] H. Liu, Y. Liu, D. Zhua, Chemical doping of graphene, J. Mater. Chem., 21 (2011) 33353345.

[54] W. S, H. Jr., R.E. Offeman, Preparation of graphitic oxide, J. Am. Chem. Soc., 80 (1958) 1339-1339.

[55] J. Zhang, F. Zhang, H. Yang, X. Huang, H. Liu, J. Zhang, S. Guo, Graphene oxide as a m atrix for enzyme immobilization, Langmuir, 26 (2010) 6083-6085

[56] J. Kim, L.J. Cote, F. Kim, W. Yuan, K.R. Shull, J. Huang, Graphene oxide sheets at interfaces, J. Am. Chem. Soc., 132 (2010) 8180-8186.

[57] M. Sprinkle, M. Ruan, Y. Hu, J. Hankinson, M. Rubio-Roy, B. Zhang, X. Wu, C. Berger, W.A. de Heer, Scalable templated growth of graphene nanoribbons on SiC, Nat. Nanotechnol., 5 (2010) 727-731.

[58] A.J. Patil, J.L. Vickery, T.B. Scott, S. Mann, Aqueous Stabilization and Self-Assembly 
of Graphene Sheets into Layered Bio-Nanocomposites using DNA, Adv. Mater., 21 (2009) 3159-3164.

[59] T.H. Han, W.J. Lee, D.H. Lee, J.E. Kim, E.Y. Choi, S.O. Kim, Peptide/graphene hybrid assembly into core/shell nanowires, Adv. Mater., 22 (2010) 2060-2064.

[60] Y. Kamiya, K. Yamazaki, T. Ogino, Protein adsorption to graphene surfaces controlled by chemical modification of the substrate surfaces, J. Colloid Interface Sci., 431 (2014) 77-81. [61] J. Katoch, S.N. Kim, Z. Kuang, B.L. Farmer, R.R. Naik, S.A. Tatulian, M. Ishigami, Structure of a peptide adsorbed on graphene and graphite, Nano Lett., 12 (2012) 2342-2346.

[62] S. Park, J. An, I. Jung, R.D. Piner, S.J. An, X. Li, A. Velamakanni, R.S. Ruoff, Colloidal suspensions of highly reduced graphene oxide in a wide variety of organic solvents, Nano Lett., 9 (2009) 1593-1597.

[63] H.C. Schniepp, J.L. Li, M.J. McAllister, H. Sai, M. Herrera-Alonso, D.H. Adamson, R.K. Prud'homme, R. Car, D.A. Saville, I.A. Aksay, Functionalized single graphene sheets derived from splitting graphite oxide, J. Phys. Chem. B, 110 (2006) 8535-8539.

[64] J. Zhang, H. Yang, G. Shen, P. Cheng, S. Guo, Reduction of graphene oxide via Lascorbic acid, Chem. Commun. (Camb), 46 (2010) 1112-1114.

[65] I. Kanayama, H. Miyaji, H. Takita, E. Nishida, M. Tsuji, B. Fugetsu, L. Sun, K. Inoue, A. Ibara, T. Akasaka, T. Sugaya, M. Kawanami, Comparative study of bioactivity of collagen scaffolds coated with graphene oxide and reduced graphene oxide, Int. J. Nanomedicine, 9 (2014) 3363-3373.

[66] Q. Liu, M. Zhang, L. Huang, Y. Li, J. Chen, C. Li, G. Shi, High-Quality Graphene Ribbons Prepared from Graphene Oxide Hydrogels and Their Application for Strain Sensors, ACS Nano, 9 (2015) 12320-12326.

[67] J.A. Hubbell, Biomaterials in tissue engineering, Nat. Biotechnol., 13 (1995) 565-576.

[68] M. Lutolf, J. Hubbell, Synthetic biomaterials as instructive extracellular microenvironments for morphogenesis in tissue engineering, Nat. biotechnol., 23 (2005) 4755.

[69] C. Berger, Z. Song, X. Li, X. Wu, N. Brown, C. Naud, D. Mayou, T. Li, J. Hass, A.N. Marchenkov, E.H. Conrad, P.N. First, W.A. de Heer, Electronic Confinement and Coherence in Patterned Epitaxial Graphene, Science, 312 (2006) 1191-1196.

[70] C.H. Lu, C.L. Zhu, J. Li, J.J. Liu, X. Chen, H.H. Yang, Using graphene to protect DNA from cleavage during cellular delivery, Chem. Commun. (Camb), 46 (2010) 3116-3118.

[71] Z. Tang, H. Wu, J.R. Cort, G.W. Buchko, Y. Zhang, Y. Shao, I.A. Aksay, J. Liu, Y. Lin, Constraint of DNA on functionalized graphene improves its biostability and specificity, Small, 6 (2010) 1205-1209.

[72] M.A. Mintzer, E.E. Simanek, Nonviral vectors for gene delivery, Chem Rev, 109 (2009) 259-302.

[73] N. Nayerossadat, T. Maedeh, P.A. Ali, Viral and nonviral delivery systems for gene delivery, Adv. Biomed. Res., 1 (2012) 27.

[74] B. Chen, M. Liu, L. Zhang, J. Huang, J. Yao, Z. Zhang, Polyethylenimine-functionalized graphene oxide as an efficient gene delivery vector, J. Mater. Chem., 21 (2011) p7736-7741.

[75] M. Kalbacova, A. Broz, J. Kong, M. Kalbac, Graphene substrates promote adherence of human osteoblasts and mesenchymal stromal cells, Carbon, 48 (2010) 4323-4329.

[76] K. Zhou, G.A. Thouas, C.C. Bernard, D.R. Nisbet, D.I. Finkelstein, D. Li, J.S. Forsythe, Method to impart electro- and biofunctionality to neural scaffolds using graphenepolyelectrolyte multilayers, ACS Appl. Mater. Interfaces, 4 (2012) 4524-4531.

[77] A. Tamayol, M. Akbari, N. Annabi, A. Paul, A. Khademhosseini, D. Juncker, Fiber-based tissue engineering: Progress, challenges, and opportunities, Biotechnol. Adv., 31 (2013) 669- 
687.

[78] A. Tamayol, M. Bahrami, Transverse permeability of fibrous porous media, Phys. Rev. E Stat. Nonlin. Soft Matter. Phys., 83 (2011) 046314.

[79] Z. Xu, H. Sun, X. Zhao, C. Gao, Ultrastrong fibers assembled from giant graphene oxide sheets, Adv. Mater., 25 (2013) 188-193.

[80] R. Jalili, S.H. Aboutalebi, D. Esrafilzadeh, R.L. Shepherd, J. Chen, S. AminorroayaYamini, K. Konstantinov, A.I. Minett, J.M. Razal, G.G. Wallace, Scalable one-step wet-s pinning of graphene fibers and yarns from liquid crystalline dispersions of graphene oxide: $t$ owards multifunctional textiles, Adv. Funct. Mater., 23 (2013) 5345-5354.

[81] R. Jalili, S.H. Aboutalebi, D. Esrafilzadeh, K. Konstantinov, S.E. Moulton, J.M. Razal, G.G. Wallace, Organic solvent-based graphene oxide liquid crystals: a facile route toward the next generation of self-assembled layer-by-layer multifunctional 3D architectures, ACS Nano, 7 (2013) 3981-3990.

[82] E. Garcia-Tunon, S. Barg, J. Franco, R. Bell, S. Eslava, E. D'Elia, R.C. Maher, F. Guitian, E. Saiz, Printing in three dimensions with graphene, Adv. Mater., 27 (2015) 1688-1693.

[83] M.K. Shin, B. Lee, S.H. Kim, J.A. Lee, G.M. Spinks, S. Gambhir, G.G. Wallace, M.E. Kozlov, R.H. Baughman, S.J. Kim, Synergistic toughening of composite fibres by selfalignment of reduced graphene oxide and carbon nanotubes, Nat. Commun., 3 (2012) 650.

[84] P.V. Kumar, N.M. Bardhan, S. Tongay, J. Wu, A.M. Belcher, J.C. Grossman, Scalable enhancement of graphene oxide properties by thermally driven phase transformation, Nat. Chem., 6 (2014) 151-158.

[85] A.E. Jakus, E.B. Secor, A.L. Rutz, S.W. Jordan, M.C. Hersam, R.N. Shah, Threedimensional printing of high-content graphene scaffolds for electronic and biomedical applications, ACS Nano, 9 (2015) 4636-4648.

[86] W.J. Rowe, Extraordinary unremitting endurance exercise and permanent injury to normal heart, Lancet, 340 (1992) 712-714.

[87] D. Zhang, I.Y. Shadrin, J. Lam, H.Q. Xian, H.R. Snodgrass, N. Bursac, Tissueengineered cardiac patch for advanced functional maturation of human ESC-derived cardiomyocytes, Biomaterials, 34 (2013) 5813-5820.

[88] R. Lakshmanan, U.M. Krishnan, S. Sethuraman, Living cardiac patch: the elixir for cardiac regeneration, Expert opin. biol.1 ther., 12 (2012) 1623-1640.

[89] J.-O. You, M. Rafat, G.J. Ye, D.T. Auguste, Nanoengineering the heart: conductive scaffolds enhance connexin 43 expression, Nano letters, 11 (2011) 3643-3648.

[90] T. Dvir, B.P. Timko, M.D. Brigham, S.R. Naik, S.S. Karajanagi, O. Levy, H. Jin, K.K. Parker, R. Langer, D.S. Kohane, Nanowired three-dimensional cardiac patches, Nat. Nanotechnol., 6 (2011) 720-725.

[91] B. Liau, D. Zhang, N. Bursac, Functional cardiac tissue engineering, Regen. Med., 7 (2012) 187-206.

[92] A. Paul, Nanocomposite hydrogels: an emerging biomimetic platform for myocardial therapy and tissue engineering, Nanomedicine (Lond), 10 (2015) 1371-1374.

[93] J. Park, S. Park, S. Ryu, S.H. Bhang, J. Kim, J.K. Yoon, Y.H. Park, S.P. Cho, S. Lee, B.H. Hong, B.S. Kim, Graphene-regulated cardiomyogenic differentiation process of mesenchymal stem cells by enhancing the expression of extracellular matrix proteins and cell signaling molecules, Adv. Healthc. Mater., 3 (2014) 176-181.

[94] E. Bressan, L. Ferroni, C. Gardin, L. Sbricoli, L. Gobbato, F.S. Ludovichetti, I. Tocco, A. Carraro, A. Piattelli, B. Zavan, Graphene based scaffolds effects on stem cells commitment, J. Transl. Med., 12 (2014) 296.

[95] J. Park, B. Kim, J. Han, J. Oh, S. Park, S. Ryu, S. Jung, J.-Y. Shin, B.S. Lee, B.H. Hong, 
D. Choi, B.-S. Kim, Graphene Oxide Flakes as a Cellular Adhesive: Prevention of Reactive Oxygen Species Mediated Death of Implanted Cells for Cardiac Repair, ACS Nano, 9 (2015) 4987-4999.

[96] J. Park, Y.S. Kim, S. Ryu, W.S. Kang, S. Park, J. Han, H.C. Jeong, B.H. Hong, Y. Ahn, B.-S. Kim, Graphene Potentiates the Myocardial Repair Efficacy of Mesenchymal Stem Cells by Stimulating the Expression of Angiogenic Growth Factors and Gap Junction Protein, Adv. Funct. Mater., 25 (2015) 2590-2600.

[97] S.R. Shin, B. Aghaei-Ghareh-Bolagh, X. Gao, M. Nikkhah, S.M. Jung, A. DolatshahiPirouz, S.B. Kim, S.M. Kim, M.R. Dokmeci, X.S. Tang, A. Khademhosseini, Layer-by-layer assembly of 3D tissue constructs with functionalized graphene, Adv. Funct. Mater., 24 (2014) 6136-6144.

[98] N. Annabi, S.R. Shin, A. Tamayol, M. Miscuglio, M.A. Bakooshli, A. Assmann, P. Mostafalu, J.Y. Sun, S. Mithieux, L. Cheung, X.S. Tang, A.S. Weiss, A. Khademhosseini, Highly Elastic and Conductive Human-Based Protein Hybrid Hydrogels, Adv. Mater., (2015). [99] M.R. Picciotto, M.J. Higley, Y.S. Mineur, Acetylcholine as a neuromodulator: cholinergic signaling shapes nervous system function and behavior, Neuron, 76 (2012) 116129.

[100] J. Bai, X. Zhong, S. Jiang, Y. Huang, X. Duan, Graphene nanomesh, Nat. Nanotechnol., 5 (2010) 190-194.

[101] G. Keller, H.R. Snodgrass, Human embryonic stem cells: the future is now, Nat. Med., 5 (1999) 151-152.

[102] D. Yang, T. Li, M. Xu, F. Gao, J. Yang, Z. Yang, W. Le, Graphene oxide promotes the differentiation of mouse embryonic stem cells to dopamine neurons, Nanomedicine (Lond), 9 (2014) 2445-2455.

[103] M.P. Lutolf, J.A. Hubbell, Synthetic biomaterials as instructive extracellular microenvironments for morphogenesis in tissue engineering, Nat. Biotechnol., 23 (2005) 4755.

[104] Y.S. Chou, T.H. Young, P.J. Lou, Effects of biomaterial-derived fibroblast conditioned medium on the alpha-amylase expression of parotid gland acinar cells, Acta Biomater., 27 (2015) 214-223.

[105] R. Meamar, L. Dehghani, M. Ghasemi, F. Khorvash, V. Shaygannejad, Stem cell therapy in stroke: a review literature, Int. J. Prev. Med., 4 (2013) S139-146.

[106] Y.C. Li, L.K. Tsai, J.H. Wang, T.H. Young, A neural stem/precursor cell monolayer for neural tissue engineering, Biomaterials, 35 (2014) 1192-1204.

[107] P. Dibajnia, C.M. Morshead, Role of neural precursor cells in promoting repair following stroke, Acta Pharmacol. Sin., 34 (2013) 78-90.

[108] S.Y. Park, J. Park, S.H. Sim, M.G. Sung, K.S. Kim, B.H. Hong, S. Hong, Enhanced differentiation of human neural stem cells into neurons on graphene, Adv. Mater., 23 (2011) H263-267.

[109] X. Li, H. Liu, X. Niu, B. Yu, Y. Fan, Q. Feng, F.Z. Cui, F. Watari, The use of carbon nanotubes to induce osteogenic differentiation of human adipose-derived MSCs in vitro and ectopic bone formation in vivo, Biomaterials, 33 (2012) 4818-4827.

[110] F. Guilak, D.M. Cohen, B.T. Estes, J.M. Gimble, W. Liedtke, C.S. Chen, Control of stem cell fate by physical interactions with the extracellular matrix, Cell Stem Cell, 5 (2009) 17-26.

[111] R.J. Pelham, Jr., Y. Wang, Cell locomotion and focal adhesions are regulated by substrate flexibility, Proc. Natl. Acad. Sci. U. S. A., 94 (1997) 13661-13665.

[112] C. Zanden, N. Hellstrom Erkenstam, T. Padel, J. Wittgenstein, J. Liu, H.G. Kuhn, Stem 
cell responses to plasma surface modified electrospun polyurethane scaffolds, Nanomedicine, 10 (2014) 949-958.

[113] Q. Tu, L. Pang, Y. Chen, Y. Zhang, R. Zhang, B. Lu, J. Wang, Effects of surface charges of graphene oxide on neuronal outgrowth and branching, Analyst, 139 (2014) 105-115.

[114] Q. Tu, L. Pang, L. Wang, Y. Zhang, R. Zhang, J. Wang, Biomimetic choline-like graphene oxide composites for neurite sprouting and outgrowth, ACS Appl. Mater. Interfaces, 5 (2013) 13188-13197.

[115] S. Bechara, L. Wadman, K.C. Popat, Electroconductive polymeric nanowire templates facilitates in vitro C17.2 neural stem cell line adhesion, proliferation and differentiation, Acta Biomater., 7 (2011) 2892-2901.

[116] F. Sher, R. Rossler, N. Brouwer, V. Balasubramaniyan, E. Boddeke, S. Copray, Differentiation of neural stem cells into oligodendrocytes: involvement of the polycomb group protein Ezh2, Stem Cells, 26 (2008) 2875-2883.

[117] D. Mortimer, Z. Pujic, T. Vaughan, A.W. Thompson, J. Feldner, I. Vetter, G.J. Goodhill, Axon guidance by growth-rate modulation, Proc. Natl. Acad. Sci. U. S. A., 107 (2010) 52025207.

[118] M. Lorenzoni, F. Brandi, S. Dante, A. Giugni, B. Torre, Simple and effective graphene laser processing for neuron patterning application, Sci. Rep., 3 (2013) 1954.

[119] A. Solanki, S.T. Chueng, P.T. Yin, R. Kappera, M. Chhowalla, K.B. Lee, Axonal alignment and enhanced neuronal differentiation of neural stem cells on graphenenanoparticle hybrid structures, Adv. Mater., 25 (2013) 5477-5482.

[120] W.C. Lee, C.H. Lim, H. Shi, L.A. Tang, Y. Wang, C.T. Lim, K.P. Loh, Origin of enhanced stem cell growth and differentiation on graphene and graphene oxide, ACS Nano, 5 (2011) 7334-7341.

[121] P.T. Yin, S. Shah, M. Chhowalla, K.B. Lee, Design, synthesis, and characterization of graphene-nanoparticle hybrid materials for bioapplications, Chem. Rev., 115 (2015) 24832531.

[122] F. Kim, L.J. Cote, J. Huang, Graphene oxide: surface activity and two-dimensional assembly, Adv. Mater., 22 (2010) 1954-1958.

[123] L.J. Cote, J. Kim, V.C. Tung, J. Luo, F. Kim, J. Huang, Graphene oxide as surfactant sheets, Pure App. Chem., 83 (2011) 95-110.

[124] T.H. Kim, S. Shah, L. Yang, P.T. Yin, M.K. Hossain, B. Conley, J.W. Choi, K.B. Lee, Controlling differentiation of adipose-derived stem cells using combinatorial graphene hybrid-pattern arrays, ACS Nano, 9 (2015) 3780-3790.

[125] E. Anghileri, S. Marconi, A. Pignatelli, P. Cifelli, M. Galie, A. Sbarbati, M. Krampera, O. Belluzzi, B. Bonetti, Neuronal differentiation potential of human adipose-derived mesenchymal stem cells, Stem Cells Dev., 17 (2008) 909-916.

[126] O. Akhavan, E. Ghaderi, Differentiation of human neural stem cells into neural networks on graphene nanogrids, J. Mater. Chem. B, 1 (2013) 6291-6301.

[127] L.H. Hess, M. Jansen, V. Maybeck, M.V. Hauf, M. Seifert, M. Stutzmann, I.D. Sharp, A. Offenhausser, J.A. Garrido, Graphene transistor arrays for recording action potentials from electrogenic cells, Adv. Mater., 23 (2011) 5045-5049, 4968.

[128] P. Fattahi, G. Yang, G. Kim, M.R. Abidian, A review of organic and inorganic biomaterials for neural interfaces, Adv. Mater., 26 (2014) 1846-1885.

[129] G.A. Silva, Neuroscience nanotechnology: progress, opportunities and challenges, Nat. Rev. Neurosci., 7 (2006) 65-74.

[130] M. Tang, Q. Song, N. Li, Z. Jiang, R. Huang, G. Cheng, Enhancement of electrical signaling in neural networks on graphene films, Biomaterials, 34 (2013) 6402-6411. 
[131] T.C. Liu, M.C. Chuang, C.Y. Chu, W.C. Huang, H.Y. Lai, C.T. Wang, W.L. Chu, S.Y. Chen, Y.Y. Chen, Implantable Graphene-based Neural Electrode Interfaces for Electrophysiology \& Neurochemistry in In Vivo Hyperacute Stroke Model, ACS Appl. Mater. Interfaces, (2015).

[132] Z.Q. Feng, T. Wang, B. Zhao, J. Li, L. Jin, Soft Graphene Nanofibers Designed for the Acceleration of Nerve Growth and Development, Adv. Mater., (2015).

[133] C.E. Schmidt, J.B. Leach, Neural tissue engineering: strategies for repair and regeneration, Annu. Rev. Biomed. Eng., 5 (2003) 293-347.

[134] H. Millesi, J. Ganglberger, A. Berger, Erfahrungen mit der Mikrochirurgie peripherer Nerven, in: W. Axhausen, D.m.D. Buck-Gramcko (Eds.) Chirurgia Plastica et Reconstructiva, Springer Berlin Heidelberg (1967), 47-55.

[135] S. Jacobson, L. Guth, An Electrophysiological Study of the Early Stages of Peripheral Nerve Regeneration, Exp. Neurol., 11 (1965) 48-60.

[136] S.W. Hong, J.H. Lee, S.H. Kang, E.Y. Hwang, Y.S. Hwang, M.H. Lee, D.W. Han, J.C. Park, Enhanced neural cell adhesion and neurite outgrowth on graphene-based biomimetic substrates, Biomed. Res. Int., 2014 (2014) 212149.

[137] M.R. Abidian, J.M. Corey, D.R. Kipke, D.C. Martin, Conducting-polymer nanotubes improve electrical properties, mechanical adhesion, neural attachment, and neurite outgrowth of neural electrodes, Small, 6 (2010) 421-429.

[138] S. Jain, A. Sharma, B. Basu, Vertical electric field stimulated neural cell functionality on porous amorphous carbon electrodes, Biomaterials, 34 (2013) 9252-9263.

[139] N. Li, Q. Zhang, S. Gao, Q. Song, R. Huang, L. Wang, L. Liu, J. Dai, M. Tang, G. Cheng, Three-dimensional graphene foam as a biocompatible and conductive scaffold for neural stem cells, Sci. Rep., 3 (2013) 1604.

[140] J.S. Cole, A. Messing, J.Q. Trojanowski, V.M. Lee, Modulation of axon diameter and neurofilaments by hypomyelinating Schwann cells in transgenic mice, J. Neurosci., 14 (1994) 6956-6966.

[141] X. Shi, H. Chang, S. Chen, C. Lai, A. Khademhosseini, H. Wu, Regulating Cellular Behavior on Few-Layer Reduced Graphene Oxide Films with Well-Controlled Reduction States, Adv. Funct. Mater., 22 (2012) 751-759.

[142] J. Venkatesan, R. Pallela, S.-K. Kim, Applications of carbon nanomaterials in bone tissue engineering, J. Biomed. Nanotechnol., 10 (2014) 3105-3123.

[143] A.J. Engler, S. Sen, H.L. Sweeney, D.E. Discher, Matrix elasticity directs stem cell lineage specification, Cell, 126 (2006) 677-689.

[144] T.R. Nayak, H. Andersen, V.S. Makam, C. Khaw, S. Bae, X. Xu, P.-L.R. Ee, J.-H. Ahn, B.H. Hong, G. Pastorin, Graphene for controlled and accelerated osteogenic differentiation of human mesenchymal stem cells, ACS nano, 5 (2011) 4670-4678.

[145] O. Akhavan, E. Ghaderi, M. Shahsavar, Graphene nanogrids for selective and fast osteogenic differentiation of human mesenchymal stem cells, Carbon, 59 (2013) 200-211.

[146] S. Kim, S.H. Ku, S.Y. Lim, J.H. Kim, C.B. Park, Graphene-biomineral hybrid materials, Adv. Mater., 23 (2011) 2009-2014.

[147] J. Lu, Y.S. He, C. Cheng, Y. Wang, L. Qiu, D. Li, D. Zou, Self-Supporting Graphene Hydrogel Film as an Experimental Platform to Evaluate the Potential of Graphene for Bone Regeneration, Adv. Funct. Mater., 23 (2013) 3494-3502.

[148] H. Xie, T. Cao, J.V. Gomes, A.H.l.C. Neto, V. Rosa, Two and three-dimensional graphene substrates to magnify osteogenic differentiation of periodontal ligament stem cells, Carbon, 93 (2015) 266-275. 
[149] J.H. Lee, Y.C. Shin, S.-M. Lee, O.S. Jin, S.H. Kang, S.W. Hong, C.-M. Jeong, J.B. Huh, D.-W. Han, Enhanced Osteogenesis by Reduced Graphene Oxide/Hydroxyapatite Nanocomposites, Sci. Rep., 5 (2015) 18833.

[150] J.H. Lee, Y.C. Shin, O.S. Jin, S.H. Kang, Y.-S. Hwang, J.-C. Park, S. Hong, D.-W. Han, Reduced graphene oxide-coated hydroxyapatite composites stimulate spontaneous osteogenic differentiation of human mesenchymal stem cells, Nanoscale, (2015).

[151] H. Liu, J. Cheng, F. Chen, F. Hou, D. Bai, P. Xi, Z. Zeng, Biomimetic and cellmediated mineralization of hydroxyapatite by carrageenan functionalized graphene oxide, ACS App Mater. Interfaces, 6 (2014) 3132-3140.

[152] X. Xie, K. Hu, D. Fang, L. Shang, S.D. Tran, M. Cerruti, Graphene and hydroxyapatite self-assemble into homogeneous, free standing nanocomposite hydrogels for bone tissue engineering, Nanoscale, 7 (2015) 7992-8002.

[153] J. Liang, Y. Liu, L. Guo, L. Li, Facile one-step synthesis of a 3D macroscopic SnO 2graphene aerogel and its application as a superior anode material for Li-ion batteries, RSC Adv., 3 (2013) 11489-11492.

[154] H.-P. Cong, X.-C. Ren, P. Wang, S.-H. Yu, Macroscopic multifunctional graphenebased hydrogels and aerogels by a metal ion induced self-assembly process, Acs Nano, 6 (2012) 2693-2703.

[155] J.H. Lee, Y.C. Shin, O.S. Jin, S.H. Kang, Y.S. Hwang, J.C. Park, S.W. Hong, D.W. Han, Reduced graphene oxide-coated hydroxyapatite composites stimulate spontaneous osteogenic differentiation of human mesenchymal stem cells, Nanoscale, 7 (2015) 11642-11651.

[156] J.H. Lee, Y.C. Shin, S.M. Lee, O.S. Jin, S.H. Kang, S.W. Hong, C.M. Jeong, J.B. Huh, D.W. Han, Enhanced Osteogenesis by Reduced Graphene Oxide/Hydroxyapatite Nanocomposites, Sci. Rep., 5 (2015) 18833.

[157] S. Kumar, K. Chatterjee, Strontium eluting graphene hybrid nanoparticles augment osteogenesis in a 3D tissue scaffold, Nanoscale, 7 (2015) 2023-2033.

[158] M. Mehrali, E. Moghaddam, S.F.S. Shirazi, S. Baradaran, M. Mehrali, S.T. Latibari, H.S.C. Metselaar, N.A. Kadri, K. Zandi, N.A.A. Osman, Synthesis, Mechanical Properties, and in Vitro Biocompatibility with Osteoblasts of Calcium Silicate-Reduced Graphene Oxide Composites, ACS App Mater. Interfaces, 6 (2014) 3947-3962.

[159] C. Gao, T. Liu, C. Shuai, S. Peng, Enhancement mechanisms of graphene in nano-58S bioactive glass scaffold: mechanical and biological performance, Sci. Rep., 4 (2014).

[160] K. Balani, R. Anderson, T. Laha, M. Andara, J. Tercero, E. Crumpler, A. Agarwal, Plasma-sprayed carbon nanotube reinforced hydroxyapatite coatings and their interaction with human osteoblasts in vitro, Biomaterials, 28 (2007) 618-624.

[161] D. Lahiri, V. Singh, A.K. Keshri, S. Seal, A. Agarwal, Carbon nanotube toughened hydroxyapatite by spark plasma sintering: microstructural evolution and multiscale tribological properties, Carbon, 48 (2010) 3103-3120.

[162] Y. Xie, H. Li, C. Zhang, X. Gu, X. Zheng, L. Huang, Graphene-reinforced calcium silicate coatings for load-bearing implants, Biomed. Mater., 9 (2014) 025009.

[163] X. Yang, L. Qiu, C. Cheng, Y. Wu, Z.F. Ma, D. Li, Ordered gelation of chemically converted graphene for next-generation electroconductive hydrogel films, Angew. Chem. Int. Ed. Engl., 50 (2011) 7325-7328.

[164] N. Dubey, R. Bentini, I. Islam, T. Cao, A.H. Castro Neto, V. Rosa, Graphene: A Versatile Carbon-Based Material for Bone Tissue Engineering, Stem Cells Int., 2015 (2015) 804213.

[165] V.C. Sanchez, A. Jachak, R.H. Hurt, A.B. Kane, Biological interactions of graphenefamily nanomaterials: an interdisciplinary review, Chem. Res. Toxicol., 25 (2011) 15-34. 
[166] S.S. Chou, M. De, J. Luo, V.M. Rotello, J. Huang, V.P. Dravid, Nanoscale graphene oxide (nGO) as artificial receptors: implications for biomolecular interactions and sensing, J. Am. Chem. Soc., 134 (2012) 16725-16733.

[167] J. Luo, L.J. Cote, V.C. Tung, A.T. Tan, P.E. Goins, J. Wu, J. Huang, Graphene oxide nanocolloids, J. Am. Chem. Soc., 132 (2010) 17667-17669.

[168] C.J. Russo, L.A. Passmore, Controlling protein adsorption on graphene for cryo-EM using low-energy hydrogen plasmas, Nat. Methods, 11 (2014) 649-652.

[169] H. Chen, M.B. Müller, K.J. Gilmore, G.G. Wallace, D. Li, Mechanically strong, electrically conductive, and biocompatible graphene paper, Adv. Mater., 20 (2008) 3557-3561. [170] J. Kim, L.J. Cote, J. Huang, Two dimensional soft material: new faces of graphene oxide, Acc. Chem. Res., 45 (2012) 1356-1364.

[171] W.-G. La, M. Jin, S. Park, H.-H. Yoon, G.-J. Jeong, S.H. Bhang, H. Park, K. Char, B.-S. Kim, Delivery of bone morphogenetic protein-2 and substance $\mathrm{P}$ using graphene oxide for bone regeneration, Int. J. Nanomedicine, 9 (2014) 107.

[172] J.A. Buckwalter, H.J. Mankin, A.J. Grodzinsky, Articular cartilage and osteoarthritis, Instr. Course Lect., 54 (2005) 465.

[173] W.C. Lee, C.H. Lim, C. Su, K.P. Loh, C.T. Lim, Cell-Assembled Graphene Biocomposite for Enhanced Chondrogenic Differentiation, Small, 11 (2015) 963-969.

[174] H.H. Yoon, S.H. Bhang, T. Kim, T. Yu, T. Hyeon, B.S. Kim, Dual Roles of Graphene Oxide in Chondrogenic Differentiation of Adult Stem Cells: Cell-Adhesion Substrate and Growth Factor-Delivery Carrier, Adv. Funct. Mater., 24 (2014) 6455-6464.

[175] J. Liao, Y. Qu, B. Chu, X. Zhang, Z. Qian, Biodegradable CSMA/PECA/Graphene Porous Hybrid Scaffold for Cartilage Tissue Engineering, Sci. Rep., 5 (2015) 9879.

[176] W.C. Lee, C.H. Lim, Kenry, C. Su, K.P. Loh, C.T. Lim, Cell-assembled graphene biocomposite for enhanced chondrogenic differentiation, Small, 11 (2015) 963-969.

[177] A.H. Lu, G.P. Hao, Q. Sun, Design of Three-Dimensional Porous Carbon Materials: From Static to Dynamic Skeletons, Angew. Chemie. Int. Ed. Engl., 52 (2013) 7930-7932.

[178] S. Ahadian, J. Ramón-Azcón, H. Chang, X. Liang, H. Kaji, H. Shiku, K. Nakajima, M. Ramalingam, H. Wu, T. Matsue, Electrically regulated differentiation of skeletal muscle cells on ultrathin graphene-based films, RSC Adv., 4 (2014) 9534-9541.

[179] P. Bajaj, J.A. Rivera, D. Marchwiany, V. Solovyeva, R. Bashir, Graphene-Based Patterning and Differentiation of C2C12 Myoblasts, Adv. Healthc. Mater., 3 (2014) 995-1000. [180] J. Mu, C. Hou, H. Wang, Y. Li, Q. Zhang, M. Zhu, Origami-inspired active graphenebased paper for programmable instant self-folding walking devices, Sci. Adv., 1 (2015) e1500533.

[181] E.J. Lee, J.H. Lee, Y.C. Shin, D.-G. Hwang, J.S. Kim, O.S. Jin, L. Jin, S.W. Hong, D.W. Han, Graphene Oxide-decorated PLGA/Collagen Hybrid Fiber Sheets for Application to Tissue Engineering Scaffolds, Biomater. Res., 18 (2014) 18-24.

[182] Z. Li, H. Wang, B. Yang, Y. Sun, R. Huo, Three-dimensional graphene foams loaded with bone marrow derived mesenchymal stem cells promote skin wound healing with reduced scarring, Mater. Sci. Eng. C Mater. Biol. Appl., 57 (2015) 181-188.

[183] J. Kim, K.S. Choi, Y. Kim, K.T. Lim, H. Seonwoo, Y. Park, D.H. Kim, P.H. Choung, C.S. Cho, S.Y. Kim, Y.H. Choung, J.H. Chung, Bioactive effects of graphene oxide cell culture substratum on structure and function of human adipose-derived stem cells, J. Biomed. Mater. Res. A, 101 (2013) 3520-3530.

[184] K. Donaldson, R. Aitken, L. Tran, V. Stone, R. Duffin, G. Forrest, A. Alexander, Carbon nanotubes: a review of their properties in relation to pulmonary toxicology and 
workplace safety, Toxicol. Sci., 92 (2006) 5-22.

[185] A. Bianco, Graphene: safe or toxic? The two faces of the medal, Angew. Chem. Int. Ed. Engl., 52 (2013) 4986-4997.

[186] H. Ren, C. Wang, J. Zhang, X. Zhou, D. Xu, J. Zheng, S. Guo, DNA cleavage system of nanosized graphene oxide sheets and copper ions, ACS Nano, 4 (2010) 7169-7174.

[187] Y. Wang, Z. Li, D. Hu, C.T. Lin, J. Li, Y. Lin, Aptamer/graphene oxide nanocomplex for in situ molecular probing in living cells, J. Am. Chem. Soc., 132 (2010) 9274-9276.

[188] P. Wilczek, R. Major, L. Lipinska, J. Lackner, A. Mzyk, Thrombogenicity and biocompatibility studies of reduced graphene oxide modified acellular pulmonary valve tissue, Mater. Sci. Eng. C Mater. Biol. Appl., 53 (2015) 310-321.

[189] X. Zhang, J. Yin, C. Peng, W. Hu, Z. Zhu, W. Li, C. Fan, Q. Huang, Distribution and biocompatibility studies of graphene oxide in mice after intravenous administration, Carbon, 49 (2011) 986-995.

[190] L. Wang, Y. Wang, T. Xu, H. Liao, C. Yao, Y. Liu, Z. Li, Z. Chen, D. Pan, L. Sun, M. $\mathrm{Wu}$, Gram-scale synthesis of single-crystalline graphene quantum dots with superior optical properties, Nat. Commun., 5 (2014) 5357.

[191] E. Murray, S. Sayyar, B.C. Thompson, G. R., D.L. Officer, G.G. Wallace, A biofriendly green route to processable, biocompatible graphene/polymer composites., RSC Adv., 5 (2015) 45284-45290.

[192] Y. Xu, Q. Wu, Y. Sun, H. Bai, G. Shi, Three-dimensional self-assembly of graphene oxide and DNA into multifunctional hydrogels, ACS Nano, 4 (2010) 7358-7362.

[193] K. Yang, J. Wan, S. Zhang, Y. Zhang, S.T. Lee, Z. Liu, In vivo pharmacokinetics, longterm biodistribution, and toxicology of PEGylated graphene in mice, ACS Nano, 5 (2011) 516-522.

[194] S.A. Sydlik, S. Jhunjhunwala, M.J. Webber, D.G. Anderson, R. Langer, In vivo compatibility of graphene oxide with differing oxidation states, ACS Nano, 9 (2015) 38663874 .

\section{Figures}




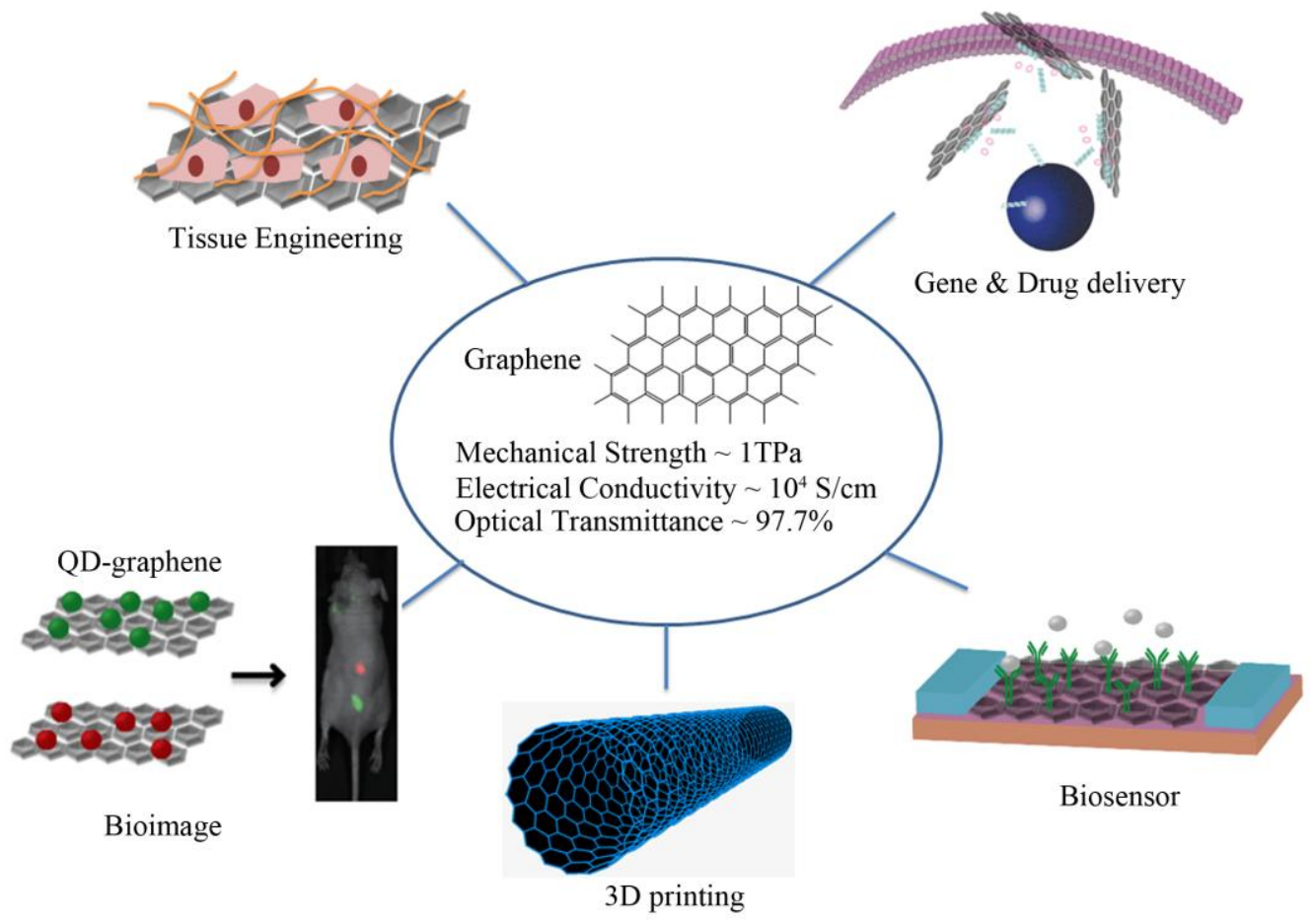

Fig.1. The overall application of graphene-based materials for regenerative medicine and tissue engineering (Reprinted with permission of bioimage from [27] Copyright (2012) American Chemical Society). 


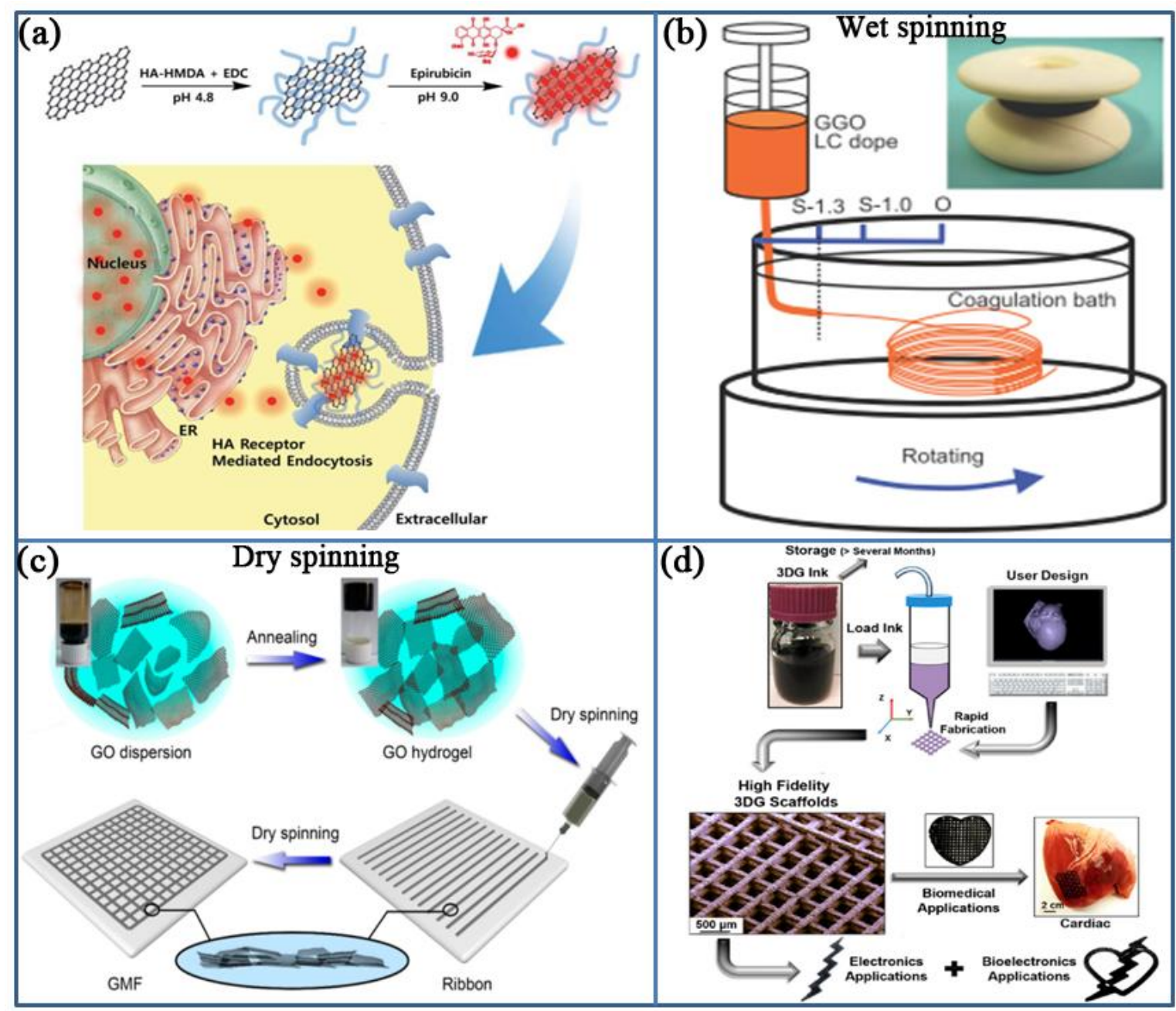

Fig. 2. (a) Schematic illustration for the preparation and released process of nano GO-based drug delivery carriers (Reprinted with permission from [26] Copyright (2014) Royal Society of Chemistry). (b) Wet-spinning: The device for wet spinning of GO fibers. (Reprinted with permission from [79] Copyright (2013) John Wiley \& Sons, Inc.). (c) Dry-spinning: schematic illustration of the gelation of a GO dispersion and the fabrication of GO ribbons and mesh fabrics (Reprinted with permission from [66] Copyright (2015) American Chemical Society). (d) Modular architectural design bases of 3D-printed graphene based inks (Reprinted with permission from [85] Copyright (2015) American Chemical Society). 


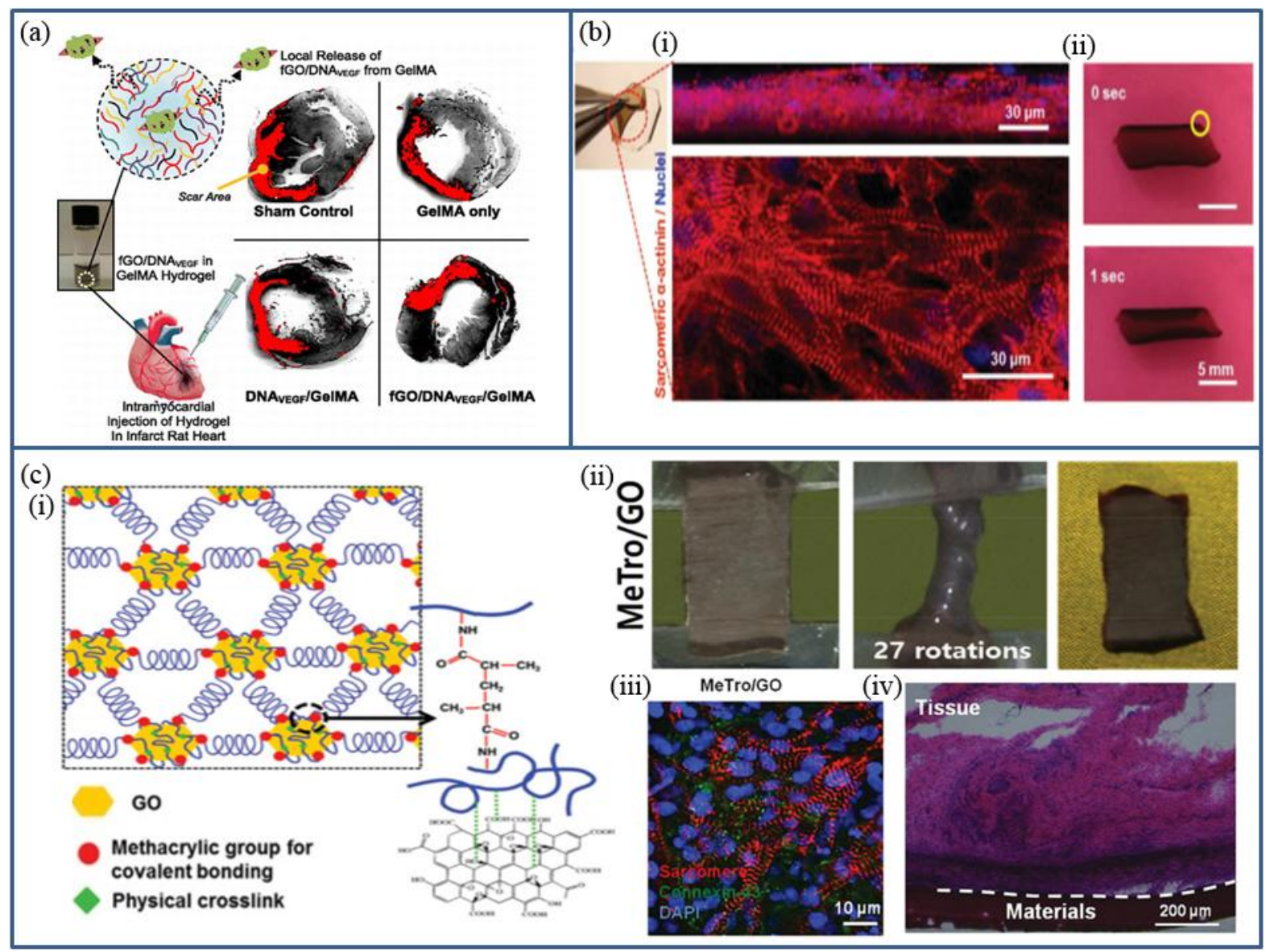

Fig. 3. Applications of graphene-based materials for cardiac tissue engineering. (a) Scar area determination by morph metric analysis. Images indicated that the cardiac fibrosis regions of left ventricle myocardial sections were stained by Sirius red. Sham operated and untreated infarcted group were used as controls. The figure showed ECM deposition (red area) in the scar tissue and the myocardium (the gray area) (Reprinted with permission from [22] Copyright (2014) American Chemical Society). (b-i) A photograph of the peeling process of cellular construct and the cross-section (top image) and top view (bottom image) confocal images of the interconnected sarcomeric structures of the 2-layer cardiomyocyte construct after 3-days of incubation. The cardiac cells were immunostained with sarcomeric $\alpha$-actinin (red) and nuclei with DAPI (blue). (b-ii) Optical images of the 2-layer cardiac actuator (Reprinted with permission from [97] Copyright (2014) John Wiley \& Sons, Inc.). (c-i) The formation of MeTro/GO network by both chemical and physical bonding. (c-ii) Images of MeTro/GO hydrogels under rotation strain (before the test (left panel), during the test (middle image), recovered gel after 10 rounds (right image)). (c-iii) Immunostaining of cardiomyoctes-seeded MeTro/GO gels after 7 days of incubation. Cells were labeled with connexin-43 (green), sarcomeric $\alpha$-actinin (red), and nuclei (blue). (c-iv) Immunohistology images of MeTro/GO hydrogels after subcutaneous implantation for 28 days. (Reprinted with permission from [98] Copyright (2015) John Wiley \& Sons, Inc.). 


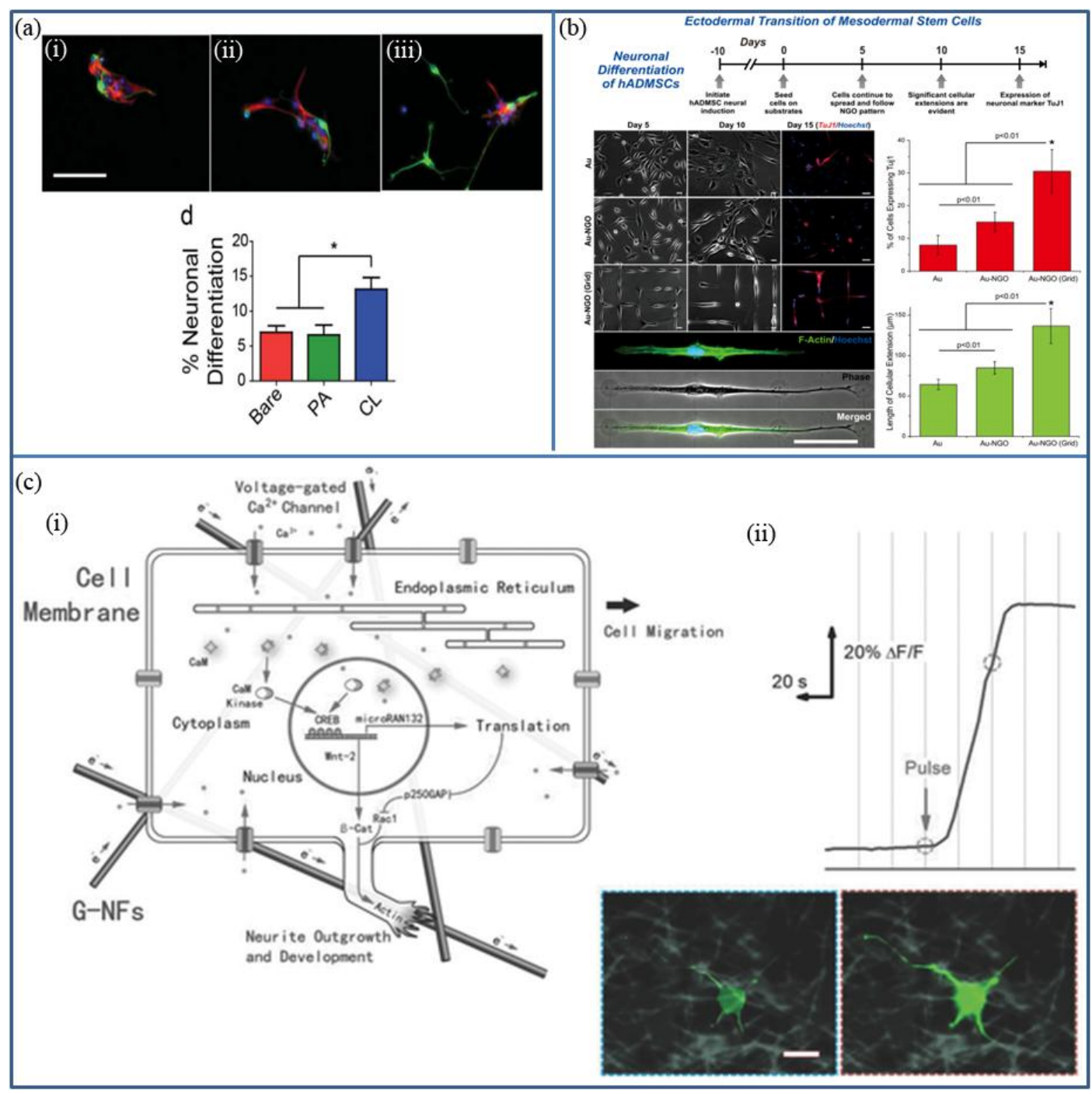

Fig. 4. Fluorescence imaging of NSCs on the (a-i) bare GO/PEDOT, (a-ii) IFN $\gamma$-coated GO/PEDOT, and (a-iii) IFN $\gamma$-crosslinked GO/PEDOT nanocomposites. Cells were labeled for glial fibrillary acidic protein (astrocyte marker, red), $\beta$-III-tubulin (neuronal marker, green), and nuclei (blue) (Reprinted with permission from [48] Copyright (2015) John Wiley \& Sons, Inc.). (b) The fluorescent images and quantification of neuronal differentiation and cellular extension of hADSCs on NGO-hybrid pattern. Cells were labeled for TUJ1 (neuronal marker, red), nuclei (blue), and F-actin (green) (Reprinted with permission from [124] Copyright (2015) American Chemical Society). (c-i) Schematic diagram of the possible gene induction pathway for depolarizing effect of the G-NFs. (c-ii) Relative fluorescent images and intensity change of the neuron $(\Delta \mathrm{F} / \mathrm{F})$ on G-NFs within a pulse period. Cells were preincubated with Fluo-4AM (Reprinted with permission from [132] Copyright (2015) John Wiley \& Sons, Inc.). 

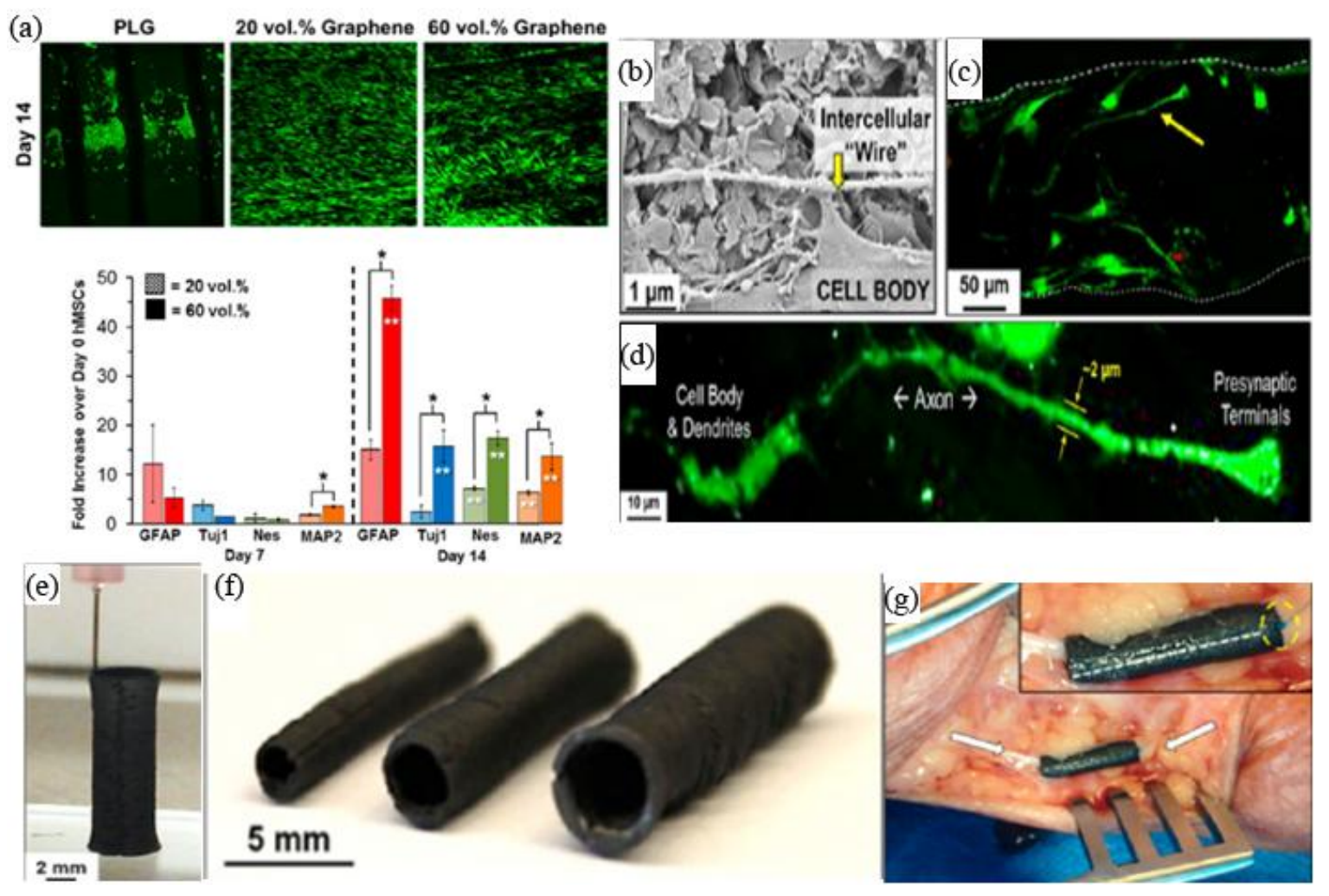

Fig. 5. (a) Optical (top row) and 3D confocal reconstruction images of live (green) and dead stained (red) hMSCs on different scaffolds for 14 days after seeding. (b) SEM micrograph of cells on $60 \mathrm{vol} \%$ graphene scaffold after 7 days of incubation. (c) 3D confocal reconstruction images of live (green) and dead stained (red) cells on 60 vol\% graphene scaffold after 14 days of incubation. (d) Yellow arrow points at the indicated cell in (b). (e) The 140-layer tubular structures of printed 3DG ink, (f) the custom-sized nerve graft conduits with various size, $(\mathrm{g})$ the photograph of 3DG nerve conduit was implanted in human cadaver (Reprinted with permission from [85] Copyright (2015) American Chemical Society). 


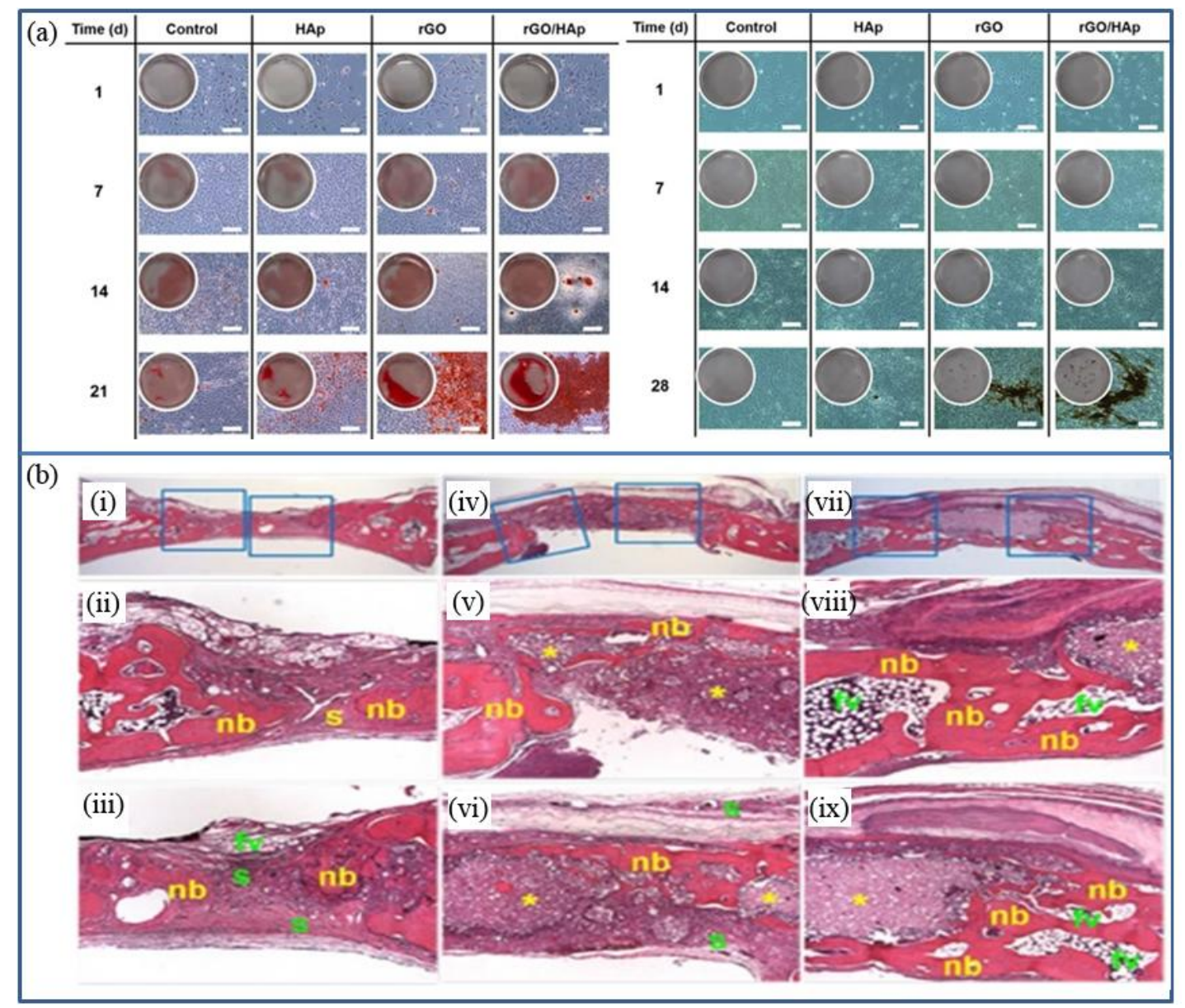

Fig. 6. (a) The Alizarin red S staining (left) and von Kossa staining (right) in MC3T3-E1 cells incubated with the HAP microparticles, rGO nanosheets or rGO/HAP nanocomposites in basal medium. Scale bar $=200 \mu \mathrm{m}$ (Reprinted with permission from [155] Copyright (2015) Royal Society of Chemistry). (b) Images from hematoxylin-eosin staining of the (i-iii) nontreated control, the minimal new bone formation in a thin, loose connective tissue originating from the defect margins and fibrovascular tissues after 4 weeks of recovery. (iv) In the group treated with the HAP grafts, dense connective tissue and small particles filled with the defect sites. (v) Minimal new bone formation, fatty marrow and fibrovascular tissues were observed. (vi) Most of the HAP microparticles was not resorbed. In the group treated with $\mathrm{rGO} / \mathrm{HAP}$ grafts, (vii) the picture of the defect site showed a larger amount of residual HAP particles related to giant cells and the inflammatory cells presented in the loose and fibrous connective tissue. (viii, ix) some new bone formation adjacent to the defect margins was observed. (Reprinted with permission from [156] Copyright (2015) nature publishing group). 


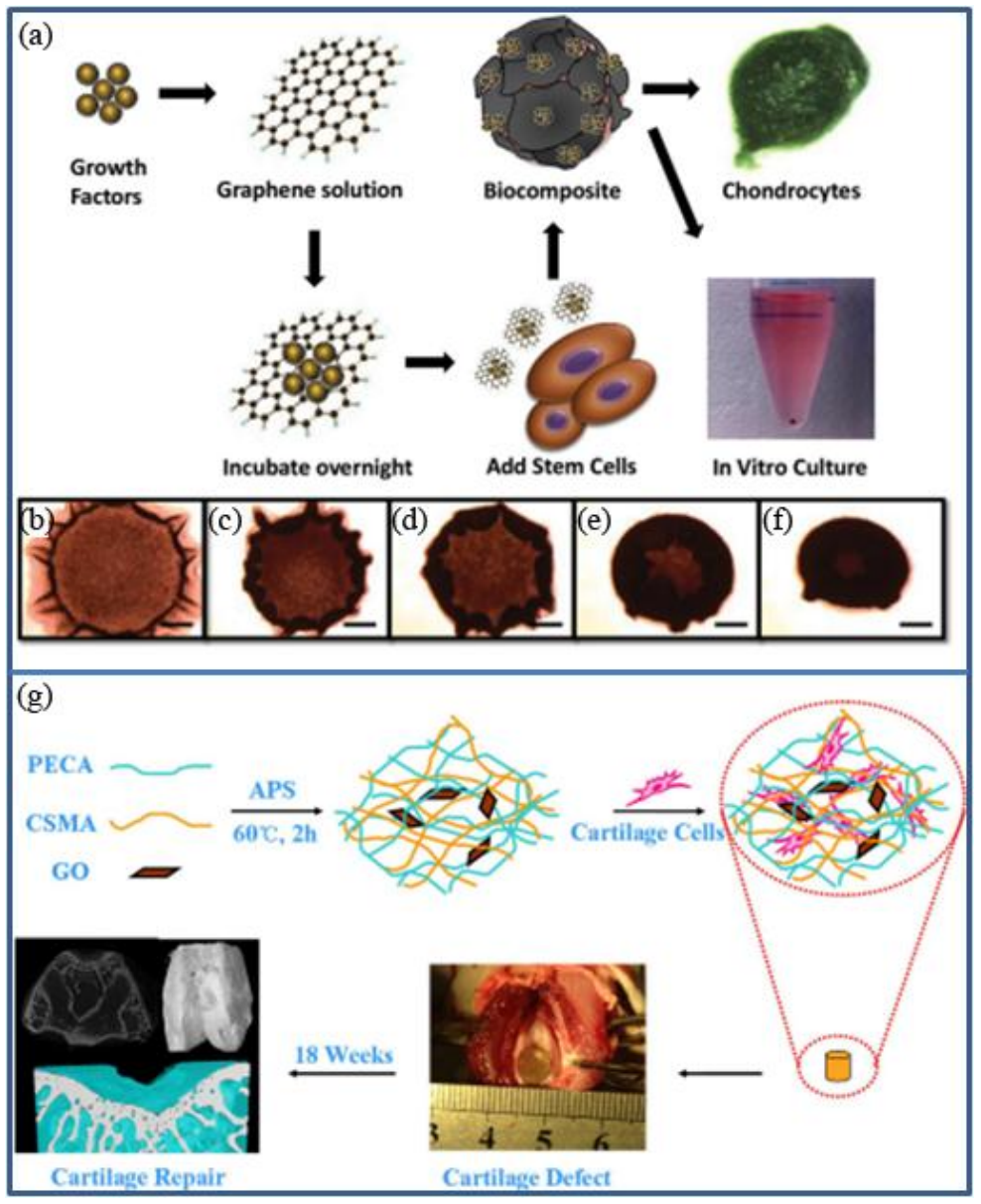

Fig.7. (a) Schematic of the design for enhancing chondrogenic differentiation via cellassembled graphene biocomposite. Graphene, GO, or porous GO (pGO) solutions preincubated with the proteins of serum and differentiated factors overnight before MSCs added. hMSCs formed into a pellet after (b) 4, (c) 8, (d) 12, (e) 20, and (f) 24 hours. Then, the medium of pellets were changed to chondrogenic induction medium for 4 weeks (Reprinted with permission from [176] Copyright (2015) John Wiley \& Sons, Inc.). (g) CSMA/PECA/GO hybrid scaffold for cartilage tissue engineering (Reprinted with permission from [175] Copyright (2015) nature publishing group). 


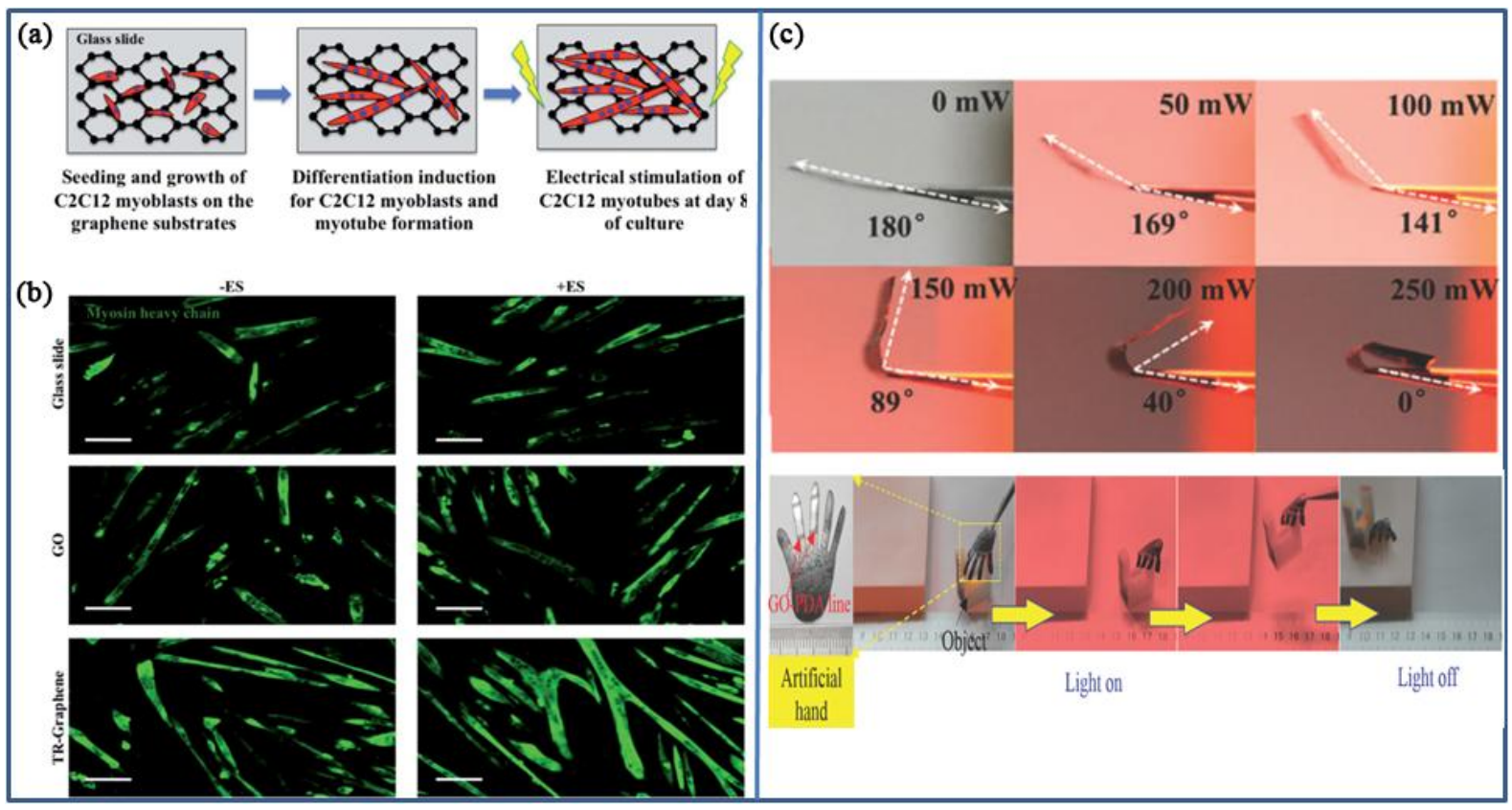

Fig.8. An applying electrical stimulation for accelerated differentiation of $\mathrm{C} 2 \mathrm{C} 12$ cells. (a) Illustration of the $\mathrm{C} 2 \mathrm{C} 12$ cells cultured on and stimulated by the graphene substrates. (b) The myosin heavy chain expressions of $\mathrm{C} 2 \mathrm{C} 12$ cells cultured on the glass, GO, and TR-graphene substrates in the absence (-ES) or presence (+ES) of applying the electrical stimulation at 10 day of incubation. Scale bars show $50 \mu \mathrm{m}$. (Reprinted with permission from [178] Copyright (2014) The Royal Society of Chemistry). (c) Dependence of bending angle on illumination intensity and optical images showing artificial/robotic hand holding an object driven by light irradiation (Reprinted with permission from [180] Copyright (2015) American Association for the Advancement of Science). 

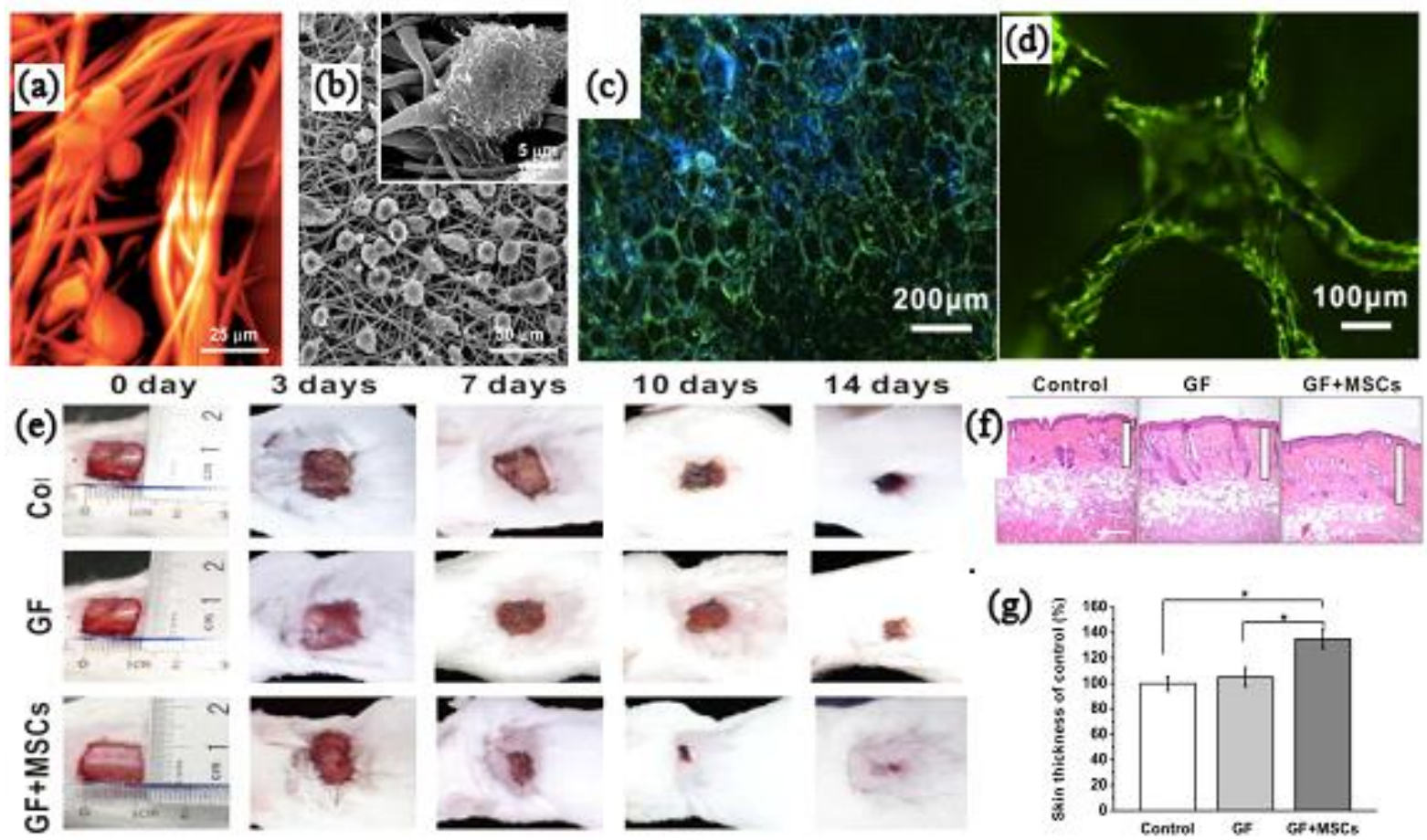

(g)

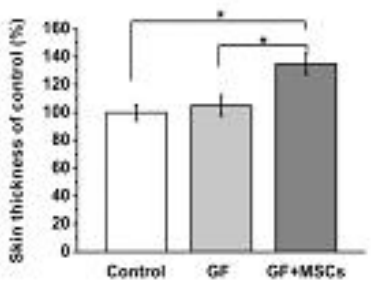

Fig.9. (a, b) Cellular behaviors of HDF on GO-PLGA/collagen fiber sheets on 5 days (Reprinted with permission from [181] Copyright (2014) Springer). (c, d) MSC growth on GFs. Representative images of MSCs on the GFs, cells were immunostained against $\beta$ tubulin (green) and the nucleus were stained by DAPI (blue). (e) Evaluation of the effect of GF and GF + MSCs over wound closure. The images of the different group wounds posttransplantation of 3, 7, 10 and 14 days. (f) Representative images of hematoxylin-eosin staining stained histological sections of the 14 days explants of different groups. Vertical white bars indicate the dermal thickness. Scale bar $=400 \mu \mathrm{m}$. (g) Quantification of skin thickness at 14 days of post-implantation in the various groups. Data were presented by means \pm SEM. ${ }^{*} \mathrm{p}<0.05$ and $\# \mathrm{p}<0.01$ (Reprinted with permission from [182] Copyright (2015) ELSEVIER). 

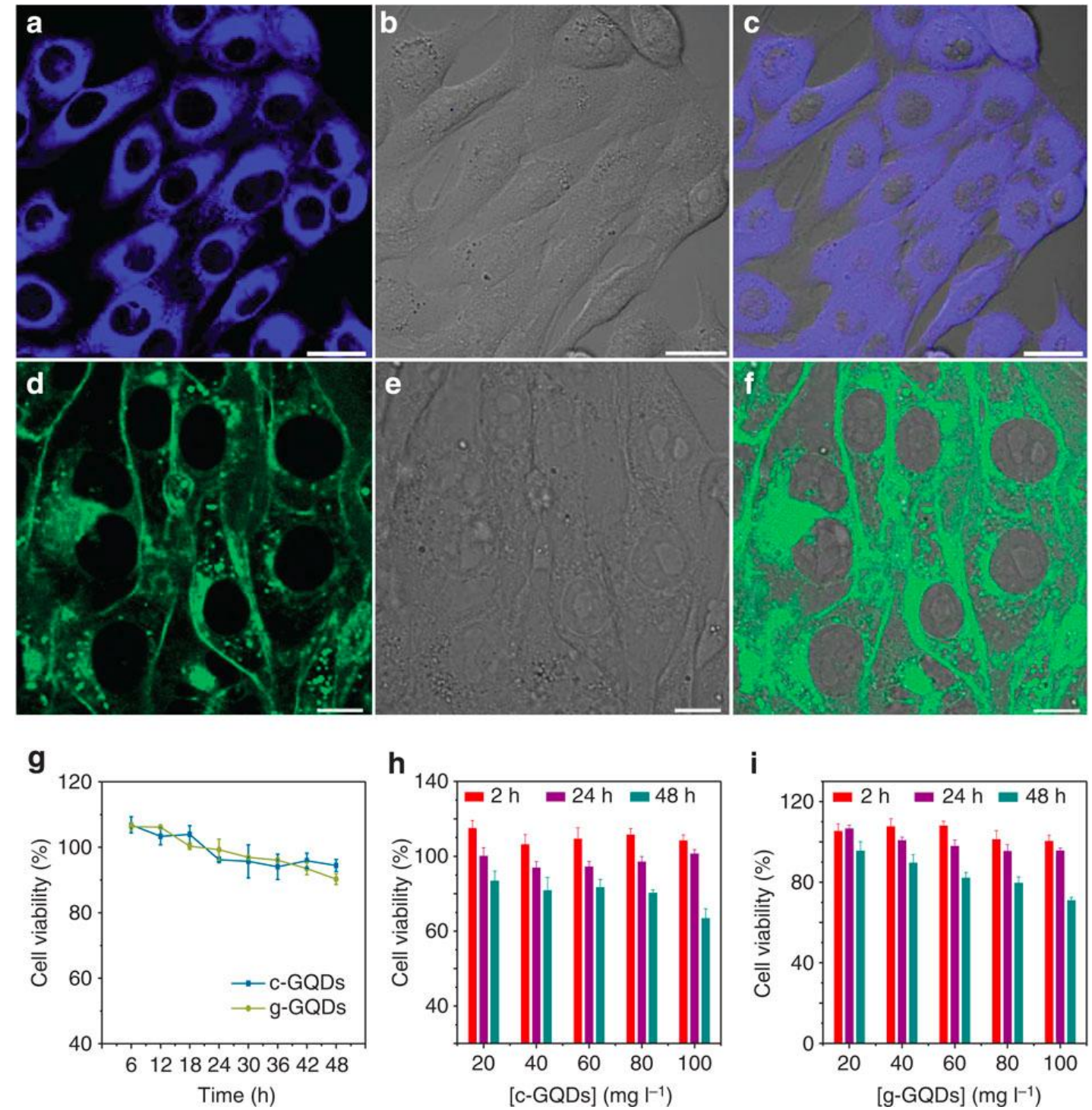

Fig. 10. Imaging and cytotoxicity estimation of GQDs using 4T1 mouse breast tumor cells (4T1 cells). (a-c) Confocal images (blue pseudocolour) at $405 \mathrm{~nm}$ excitation of 4T1 cells using cyan fluorescent GQDs (c-GQD) internalized into the cellular cytoplasm. Scale bar = $20 \mu \mathrm{m}$. (d-f) Image of 4T1 cells labeled with green fluorescent GQDs (g-GQDs). Scale bar = $10 \mu \mathrm{m}$. (g) Viability assays of cells treated with c-GQDs and g-GQDs at the dose $(10 \mathrm{mg} / \mathrm{l})$ for culture time varied from 6 to 48 hours. Viability assays of cells treated with higher doses (h) c-GQDs and (i) g-GQDs showed the dependence of the cellular viability on GQD concentration (Reprinted with permission from [190] Copyright (2015) nature publishing group). 
(a)

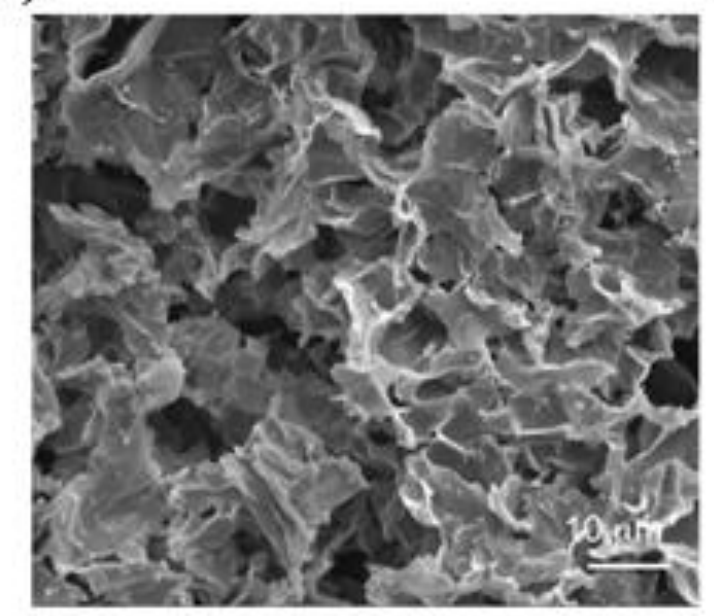

(b)

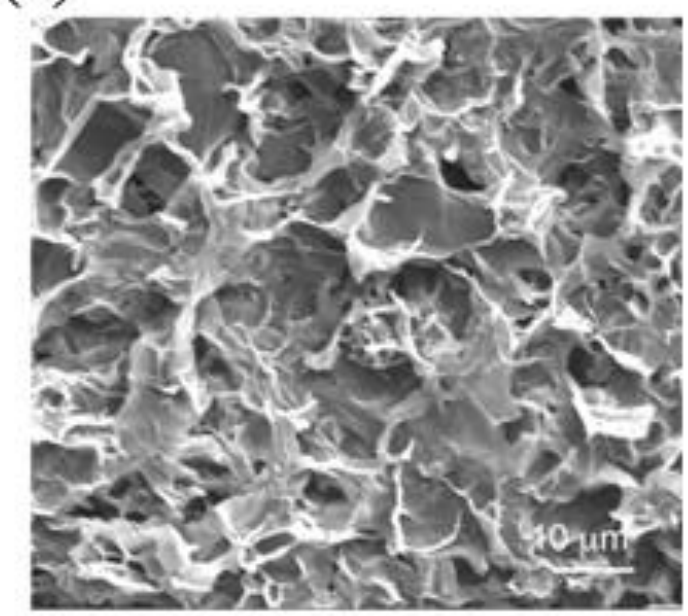

GO-R

(c)

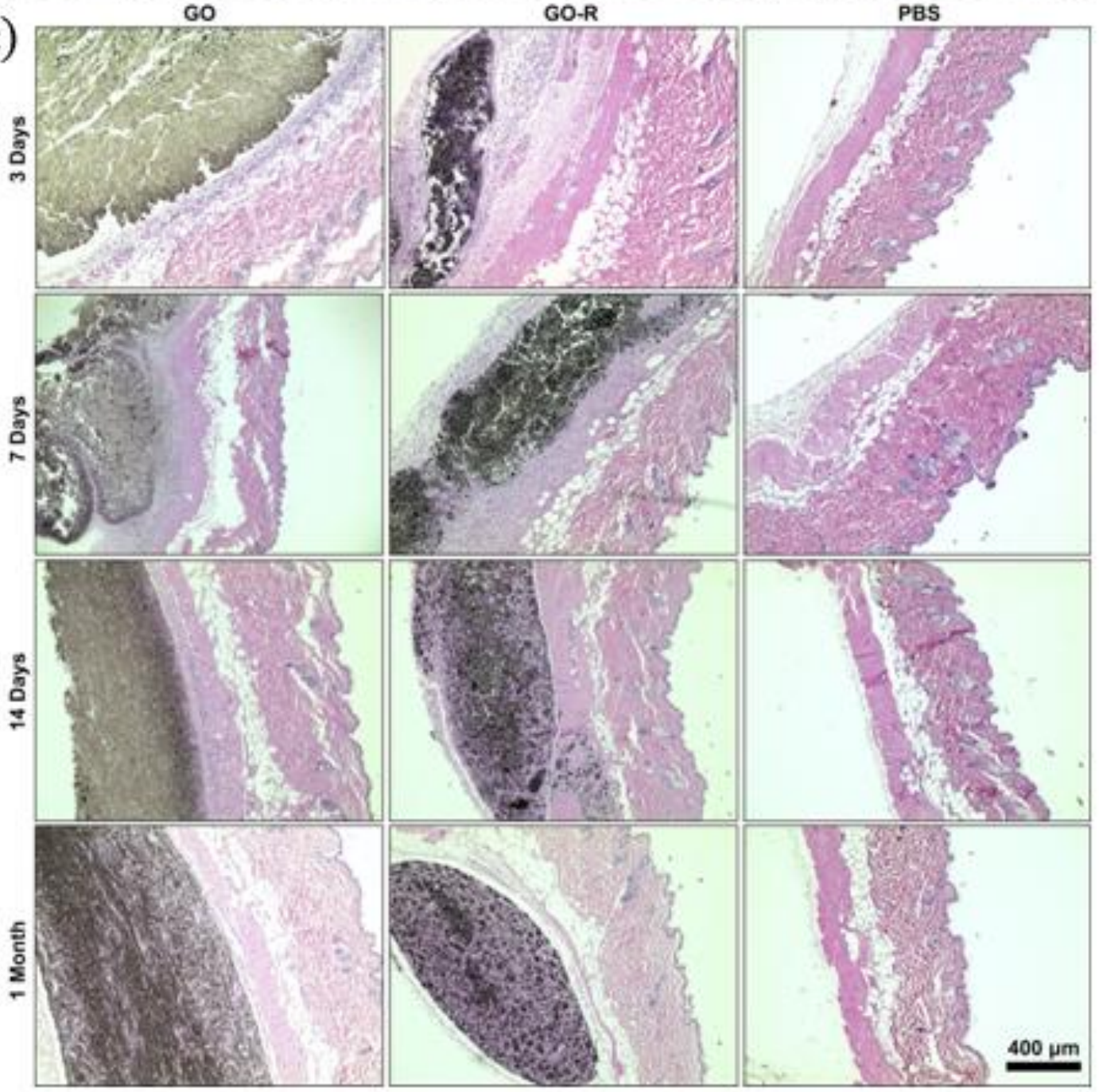

Fig.11. SEM images of GO with different $C$ to $O$ ratio in dry state, (a) GO (2.8:1) and (b) GO-R (3.1:1). (c) Hematoxylin-eosin staining of the subcutaneous tissue. GO (left), GO-R (middle), and PBS (right) at 3, 7, 14 days, and 1 month (Reprinted with permission from [194] Copyright (2015) American Chemical Society). 


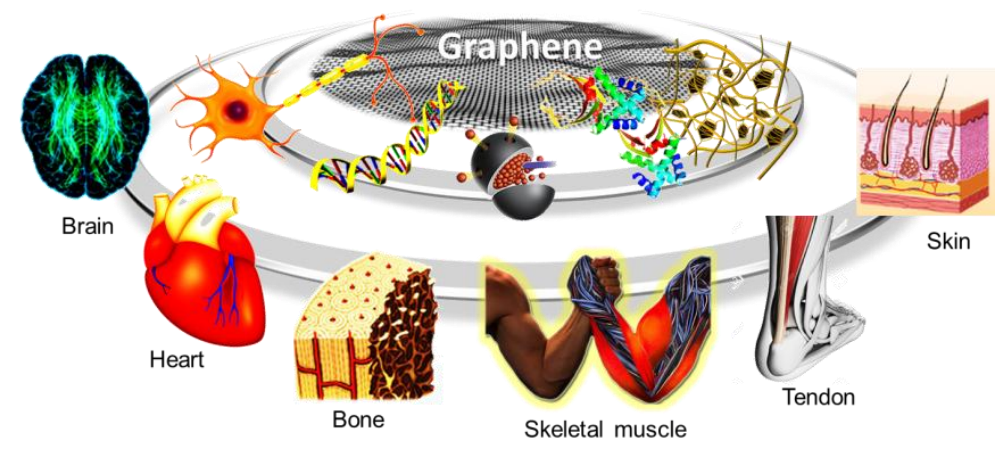

